\title{
A time-dependent freight tour synthesis model
}

\author{
Iván Sánchez-Díaz ${ }^{a, *}$, José Holguín-Veras ${ }^{b}$, Xuegang (Jeff) Ban ${ }^{c}$ \\ a Department of Technology Management and Economics, Chalmers University of Technology, Room 3334, Vera Sandbergs Allé 8, Göteborg 412 96, Sweden \\ ${ }^{\mathrm{b}}$ Department of Civil and Environmental Engineering, Rensselaer Polytechnic Institute, Room JEC 4030, 110 8th St., Troy, NY 12180, United States \\ ${ }^{\mathrm{c}}$ Department of Civil and Environmental Engineering, Rensselaer Polytechnic Institute, Room JEC 4034, 110 8th St., Troy, NY 12180, United States
}

\section{A R T I C L E I N F O}

\section{Article history:}

Received 18 April 2014

Received in revised form 21 April 2015

Accepted 22 April 2015

\section{Keywords:}

Freight demand model

OD synthesis

Multi-attribute value function

Freight tours

Urban freight

Urban freight data

\begin{abstract}
A B S T R A C T
This paper introduces a model of urban freight demand that seeks to estimate tour flows from secondary data sources e.g., traffic counts, to bypass the need for expensive surveys. The model discussed in this paper, referred as Freight Tour Synthesis (FTS), enhances current techniques by incorporating the time-dependent tour-based behavior of freight vehicles, and the decision maker's (e.g., metropolitan planning agency planner) preferences for different sources of information. The model, based on entropy maximization theory, estimates the most likely set of tour flows, given a set of freight trip generation estimates, a set of traffic counts per time interval, and total freight transportation cost in the network. The type of inputs used allows the assessment of changes in infrastructure, policy and land use. The ability of the model to replicate actual values is assessed using the Denver Region (CO) as a case study.
\end{abstract}

(c) 2015 Elsevier Ltd. All rights reserved.

\section{Introduction}

Due to the globalization process that has defined the world economy in the last several decades, most of the goods consumed in urban areas are produced miles from where they are consumed. The proliferation of commercial transactions between regions has allowed communities with some resource shortage to obtain it through exchange relationships with some other communities that have a surplus of this resource. These exchange relationships have enhanced economic development because, when two such communities are joined by trade, scarcities that were local to one or the other no longer have to be limiting (Catton, 1982). This "scope-enlargement" explains why freight transportation is intrinsic to economic development. In essence, vibrant economies require good transportation systems. The freight traffic observed in urban areas is a complex mix of different layers of economic interactions: flows of products from producing regions outside the urban area; local distribution of supplies; cargo flows between the manufacturers located in the metropolitan area; and the cargo that comes through, and is distributed from, international gateways such as ports and airports. Analyzing such an intricate web of goods movements is made more complicated by the fact that the goods are not transported directly from the primary source to retail stores, as there are intermediary processes to consider. For instance, goods can be produced in manufacturing districts, or transported to distribution centers, stored in warehouses, or transported elsewhere for further processing. Although the dynamics driving freight demand are not fully understood, planning agencies use models to replicate the distribution processes to identify infrastructure needs, manage travel demand, and formulate policies aimed at improving distribution efficiency.

\footnotetext{
* Corresponding author.

E-mail addresses: ivan.sanchez@chalmers.se (I. Sánchez-Díaz), jhv@rpi.edu (J. Holguín-Veras), banx@rpi.edu (X.J. Ban).
} 
One of the most important input data in transportation planning are Origin-Destination (OD) matrices, as they capture travel patterns, such as origin and destination of cargo and/or freight trips, their geographical distribution and their linkages to travel impedance. Traditionally, these matrices are obtained through primary data collection efforts such as surveys, and then used to calibrate trip distribution models. Although the large costs associated with primary data collection-\$10 million for a city like New York (Holguín-Veras et al., 2010)-have deterred transportation agencies from conducting large scale data collection efforts, the rise of technology in the last decades has brought to the scene ready-to-use data from secondary sources, such as traffic counts, and with it a new generation of models often referred as Origin Destination Synthesis (ODS).

The fundamental objective of ODS models is to produce trip matrices using secondary data such as traffic counts as the key input. The ODS problem is, mathematically, underspecified because the number of independent traffic counts is generally smaller than the number of OD pairs which are the unknowns. The problem can be solved using two main approaches: structured approaches and unstructured approaches (Willumsen, 1978; Ortúzar and Willumsen, 2011). Structured approaches restrict the feasible space by imposing a model structure, such as a gravity model, on the OD flows, and estimate the best parameters subject to traffic counts constraints. The main limitation of this approach is that by imposing a model structure, some information contained in the traffic counts is lost (Van Zuylen and Willumsen, 1980). In contrast, unstructured approaches seek to find a unique solution based on the traffic counts by introducing the minimum external information. The most common techniques are maximum likelihood, information minimization and entropy maximization.

Freight ODS models are called to play an important role in the development of urban freight demand models, as they have a great potential to model urban freight flows while complying with the budget restrictions of freight planning. The experience with passenger ODS models suggests their potential to identify critical infrastructure needs, evaluate performance measures, assess freight related policies, cross-check and/or update old OD matrices, and develop urban freight demand models, among others (Willumsen, 1978; Ortúzar and Willumsen, 2011). However, the vast majority of the freight ODS tend to replicate the efforts made on the passenger side without considering the particularities of goods movements' patterns. The main goal of this paper is to help address this situation.

This paper focuses on a more general version of the traditional freight ODS problem. Here, instead of simply estimating the freight vehicle flows from an origin to a destination, the model estimates the freight vehicle flows that traverse a sequence of pick-up and delivery stops. The model is referred to as Freight Tour Synthesis (FTS) model. It is important to distinguish FTS from freight ODS because they are not equivalent. While the output of freight ODS can be conveniently mapped into a two dimensional matrix, the output of FTS cannot. Essentially, it is incorrect to refer to FTS as a case of freight ODS. In fact, freight ODS is a particular case of FTS when all tours only have one stop. The model discussed in this paper follows similar principles to ODS but incorporates two key aspects of urban goods distribution: the temporality of flows and the tour-based behavior of freight vehicles.

Temporal effects are an important aspect of freight activity. The dynamic nature of urban freight transportation involves variations according to the season, the day of the week, and the hours within the day. The latter is of great importance to this paper. The fluctuation of demand over the day, as opposed to the static nature of supply, is the source of inefficiencies in transportation systems. In general, infrastructure systems are underutilized most of the day and overwhelmed during the peak hours. Variations of demand and the corresponding traffic impacts throughout the day are, therefore, key information for efficient infrastructure decision-making and demand management strategies. This temporal aspect introduces both challenges and opportunities for modeling, as considering temporal effects leads to complex models that require larger amounts of data, though the more realistic models could lead to better forecasts.

In urban areas, the distribution of goods requires complex delivery/pick-up tours that could have dozens of stops, as in the case of parcel deliveries. This reflects the fact that it is generally inefficient for suppliers to assign one vehicle to each delivery; using delivery tours is preferable when shipment sizes are small in relation to the capacity of the truck. Not surprisingly, the available data indicate that more than $80 \%$ of urban deliveries are made as part of tours with multiple stops that, depending on the city, could average from $1.80 \mathrm{stops} /$ tour (Schiedam, The Netherlands) (Vleugel and Janic, 2004) to 15.70 stops/tour (New York City, USA) (Holguín-Veras et al., 2006). The large number of stops of urban freight vehicles has important modeling implications. Modeling urban freight demand with the assumption that the individual trips in a tour are independent will inevitably produce major errors, and increase the risk of erroneous investment and policy decisions. First, the behavioral determinants that drive the tour formation process are ultimately related to routing efficiency considerations. Decomposing tours into individual trips destroys any possibility of capturing these effects. Second, assuming separate trips unavoidably forces the model to assume that the trip's explanatory variables are related to the trip's origin and destination. This is erroneous because, in reality, the physical origins and destinations of the individual trips are the result of a logistical decision that is unrelated to the generation of the flow of the cargo.

In order to help fill this void, this paper proposes an urban FTS model that explicitly considers freight vehicle tours and time-dependent effects. The paper is organized in six sections in addition to the introduction: Section 2 reviews the literature related to tour models and freight ODS models. Section 3 describes the methodology and the mathematical derivations; while Section 4 presents the application of multi-attribute value functions to the Time-Dependent Freight Tour Synthesis (TD-FTS) model. Section 5 describes the application of the model, and Section 6 presents the results obtained from the application of the FTS model to the Denver Region. Section 7 summarizes the conclusions of the paper. 


\section{Literature review}

This literature review is organized in two sections. The first section reviews the literature on tour models, and the second reviews the literature on freight ODS.

\subsection{Freight Tour Models}

The literature on (aggregate) freight demand modeling that explicitly considers tours is very sparse. A number of approaches have been proposed. These formulations can be roughly classified into 3 categories: simulation, hybrid, and analytical models (Holguín-Veras et al., 2013). Among the simulation models, one could list Boerkamps and Binsbergen (1999), Liedtke and Schepperle (2004), Stefan et al. (2005), Liedtke (2006), Hunt and Stefan (2007), Routhier and Toilier (2007), and Liedtke (2009). Stefan et al. (2005) and Hunt and Stefan (2007) propose an agent-based microsimulation to model commercial vehicles in Calgary, Canada. In their model, tour sequences are constructed using a "rubber-banding" approach, and a fleet-allocator simulation model. Routhier and Toilier (2007) develop a simulation model (FRETURB) to reproduce tour patterns observed on the area of study. These patterns are obtained from a large scale urban freight survey that is regularly conducted in France. Their model has been successfully applied to a number of French cities. Boerkamps and Binsbergen (1999) develop the GoodTrip model to study urban freight distribution in Groningen, The Netherlands. The GoodTrip estimates commodity flows according to the spatial distribution of activities, and convert these flows to vehicle tour flows using micro-simulations based on the origin's activity type. Liedtke and Schepperle (2004) and Liedtke (2009) propose an actor-based approach to model freight movements. This model (InterLOG) generates vehicle tour flows based on estimated commodity flows between companies and a market simulation module.

Although simulation models have proven very useful in describing existing conditions, an added analytical component provides a robust foundation for modeling purposes, particularly, if the analytical component is supported by proper behavioral and/or economic axioms. This type of models can be referred to as hybrids. For instance, Wisetjindawat et al. (2007) use a commodity-based model to estimate production, consumption, and distribution in Tokyo, and then convert the commodity flows into vehicle flows using a fleet allocator simulation, and subsequent vehicle routing problem (VRP) simulations. van Duin et al. (2007) study auctions in the spot market, and how they affect tours structure. A simulation model assigns the loads from the spot market to each carrier, and tours are determined using a VRP with time windows. Donnelly (2007) uses a simulation model to fuse data from different sources and obtain aggregate commodity flows. Deliveries are then consolidated using a shipment assignment module based on Monte Carlo simulations and a VRP algorithm. Silas and Holguín-Veras (2009) and Holguín-Veras and Aros-Vera (2014) use a behavioral micro-simulation that reproduces the joint decision of carrier and receivers to assess the effectiveness of an Off-Hour Deliveries program in New York City.

Other models determine freight tours using analytical procedures based on behavioral, economic or statistical axioms. For instance, Holguín-Veras (2000), Thorson (2005), and Xu and Holguín-Veras (2008) use economic principles to find the commodity and vehicle tour flows that meet freight market equilibrium conditions. These models use a bi-level formulation that relates the suppliers' decisions (e.g., production level, delivery routes and profit margin) to a market competition environment. Given the combinatorial nature of these formulations, solving them is computationally challenging, and only small instances can be solved at optimality using state-of-the-art algorithms. Wang and Holguín-Veras (2008a, 2008b) generate tour sequences using a discrete choice model, in which the selection of each tour's home base depends on the location of logistical terminals; the next destination depends on the distance to the transportation analysis zones (TAZ), the amount of cargo available for pickup at the destination, the amount of cargo available for delivery at the destination, and the number of available destinations at the same stratum; while the termination of the tour depends on the distance to the home base, the cumulative distance covered up to the current location and the cumulative amount of cargo picked up until the current location. Although these analytical approaches have a strong behavioral foundation, they require a significant amount of data and computational capacity.

Other analytical models are based on statistical axioms, such as entropy maximization. Although not a tour model, the work of Wilson (1969a, 1969b) is worth of specific mention as he developed the entropy-based concept used in this research, including the gravity model, to estimate trip flows between TAZ. This work is one of the pillars of the classical four steps model widely used for urban transportation systems planning. The basic model maximizes an entropy function of flows, subject to attraction, production, and total cost constraints. The Lagrange multipliers resulting from the calibration process can, then, be used as parameters to estimate aggregate trip flows between TAZs, as follows:

$$
x_{i j}=\exp \left(\lambda_{i} O_{i}+\lambda_{j} D_{j}+\beta c_{i j}\right)
$$

where the vehicles flow between two TAZs $\left(x_{i j}\right)$ is a function of the attraction and production of each TAZ $\left(O_{i}, D_{j}\right)$, the transportation cost between TAZs $\left(c_{i j}\right)$ and the corresponding Lagrange multipliers $\left(\lambda_{i}, \lambda_{j}\right.$ and $\left.\beta\right)$. As an extension, Wilson (1970) developed a model that distributes inter-regional commodity flows among TAZs using the same principle.

The tour model developed by Wang and Holguín-Veras (2009) is equivalent to the traditional gravity model, but instead of estimating flows from an origin to a destination, it estimates flows through a tour covering multiple stops. The model also uses entropy maximization theory, and seeks to find the most probable tour flows that respect the trip-end constraints for each TAZ, and the total impedance to travel in the network. Similarly to the gravity model, the parameters resulting from the calibration can be used to obtain tour flows using the following equation: 


$$
x_{m}^{*}=\exp \left(\sum_{i=1}^{N} \lambda_{i}^{*} O_{i m}+\beta_{1}^{*} c_{T m}+\beta_{2}^{*} c_{H m}\right)
$$

where the truck flow $\left(x_{m}^{*}\right)$ is a function of the trip-ends of each TAZ in the tour $\left(O_{i m}\right)$, the tour travel time $\left(c_{T m}\right)$, the tour handling time $\left(c_{H m}\right)$, and the corresponding Lagrange multipliers $\left(\lambda_{i}^{*}, \beta_{1}^{*}\right.$ and $\left.\beta_{2}^{*}\right)$.

This aggregate tour-based model was successfully applied to the city of Denver, Colorado. The model outperformed the traditional gravity model, and was able to replicate the observed tour flows with low errors and reproduce very closely the observed tour length distribution. In this paper, the model developed by Wilson (1969a, 1969b) is referred to as Gravity Model (GM), and the model developed by Wang and Holguín-Veras (2009) is referred to as the Static Entropy Maximization (S-EM) model, both are used as benchmark models. The TD-FTS model introduced in this paper builds on the theory developed by Wilson (1969a, 1969b) and Wang and Holguín-Veras (2009), and incorporates the principles of freight ODS to estimate tour flows.

\subsection{Freight origin-destination synthesis}

ODS was originally developed to estimate passenger demand; its fundamental objective is to produce trip matrices using traffic counts as input (Robillard, 1975). Although there is a significant amount of passenger related publications on this topic-see (Yang et al., 1992; Bera and Rao, 2011) for a detailed literature review-this is not the case for freight transportation where only a handful of papers have been published (Tamin and Willumsen, 1992; Gédéon et al., 1993; List and Turnquist, 1994a, 1994b; Tavasszy et al., 1994; Al-Battaineh and Kaysi, 2005a, 2005b; Holguín-Veras and Patil, 2007; Holguín-Veras and Patil, 2008).

The first formulation of freight ODS is that of Tamin and Willumsen (1988), who adapted a passenger ODS model to freight. The model used traffic counts and freight generation as input. The authors aimed to replicate the underlying movement patterns through functions (i.e., least squares, maximum likelihood) relating observed traffic counts to link flows estimated using a gravity model and/or a gravity opportunity model. Their main finding was that using traffic counts to estimate freight demand models turned out to be only marginally less accurate than using an observed OD matrix. However, the model does not consider empty trips, which is bound to produce major errors in directional traffic estimation (Holguín-Veras and Patil, 2007). Gedeon et al. (1993) developed an ODS formulation to obtain least cost shipments and to identify the amounts to be ordered from each supplier to be delivered to each demand center. List and Turnquist (1994a, 1994b) proposed an unstructured multi-class truck trip matrices estimation method, based on partial and fragmentary data. The authors estimated the OD matrix through a large-scale linear programming problem in which the objective is to minimize the weighted sum of all deviations from the observed values. The model was tested in New York City and then extended to border crossings in Nozick et al. (1996). Another application to international border crossings in North-Western Europe was conducted by Tavasszy et al. (1994) who used a Furness algorithm to estimate the parameters of the gravity model. Al-Battaineh and Kaysi (2005a, 2005b) developed an ODS formulation with two sub-models, the first is an input-output model that obtains the total zonal attractions and productions for different commodities. The second, based on a genetic algorithm, searches the optimum OD matrix reproducing total zonal generation and link counts on the network.

The main deficiencies of these worthy attempts are that they do not consider delivery tours and the empty trips resulting from the distribution process. However, empty trips are an unavoidable consequence of freight transportation and ignoring them is bound to produce major errors in the estimation of OD matrices, as the proportion of empty trips is usually around 20\% in urban areas and 30-40\% in inter-city conditions (Holguín-Veras and Thorson, 2000). Holguín-Veras and Patil (2007, 2008) attempted to deal with these issues.

Holguín-Veras and Patil (2007) developed three variants of an ODS model to obtain OD matrices and traffic volumes while accounting for the empty trips. The inputs for these models were traffic counts and the amount of cargo produced and attracted by each TAZ. The authors used a double constrained gravity model for trip distribution, the Noortman and van Es model (Noortman and van Es, 1978) to model empty trips, and a proportional route choice model to assign the traffic. The results showed that considering empty trips significantly reduced the errors associated with the estimation of link traffic volumes and modeling parameters. This ODS model was extended to the multi-commodity case by Holguín-Veras and Patil (2008). The comparison between the results from the multi-commodity ODS and the single commodity ODS discussed previously indicated that incorporating multiple commodities in the formulation brings about substantial reductions in the error associated with the estimation of observed traffic counts. In fact, errors decreased in the order of $20 \%$ for empty traffic and $40 \%$ for loaded and total traffic. The results also showed some minor improvements in the ability of the multi-commodity ODS formulation to estimate the OD matrices.

A more recent research attempted to exploit available technologies and data for freight ODS. Ma et al. (2012) gathered traffic information from different sources (loop detectors, cameras, Bluetooth scanners and weigh-in-motion systems) and updated a priori freight OD matrix using Bayesian Networks. Although the case study resulted in accurate OD flows estimates, this approach has two limitations: it requires a previously estimated OD matrix and the scope is limited to corridors with high density of monitoring devices, as the authors found that non-full coverage may lead to large errors.

As shown in this review, there are a number of freight specific characteristics not considered yet, corroborating that there is a large room to improve freight ODS models. In particular, the review identified two main gaps, the lack of models considering time-dependency, and tour-based behavior. This paper proposes a TD-FTS model to address these methodological gaps. 


\section{Bi-criteria freight tour synthesis model}

This section presents the methodology proposed for the TD-FTS. It is important to start by explaining the distinction between the interrelated concepts of tour sequence and tour flows. A tour sequence is an ordered listing of the nodes that are visited as part of a generic tour $m$ which starts and ends at the home base: $S_{m}=\left[n_{1}, n_{2}, \ldots, n_{m}, n_{1}\right]$; while the tour flow $x_{m}$ represents the number of vehicles that traverse the node sequence that defines the tour $m$ (Wang and Holguín-Veras, 2008a, 2008b). This distinction is of great importance because, since this is an aggregate model that focuses on depicting travel to/from TAZs, it is very likely that numerous vehicles follow the same sequence, as the nodes are defined in terms of geographic areas (the TAZs) that contain significant numbers of commercial establishments. The TD-FTS model uses Entropy Maximization Theory (Wilson, 1969a, 1969b; Wilson, 1970). The key assumption of entropy maximization is that "all possible states of the system are equally probable; that is, there is no knowledge to say that one is more probable than the others. Then the most probable estimate of a flow is that which is produced by the greatest number of system states [...] and satisfies all constraints" (Wilson, 1970). Although in the original model proposed by Wilson (1969a, 1969b) the state variables are defined as the flows on origin-destination pairs, the S-EM model introduced by (Wang and Holguín-Veras, 2008) developed an alternative definition able to consider a tour structure, which is more appropriate for freight. The TD-FTS uses these system state variables and enhances the model by considering the temporal aspect of flows, by using trip generation estimates by industry sectors and by incorporating data from traffic counts and Global Positioning Systems (GPS) to enhance the parameters calibration.

One way to incorporate traffic counts is to include them as constraints to be met. However, doing so will impose an additional parameter for traffic counts, which limits the model to one that reproduces the flows patterns under specific conditions and that are very sensitive to the input values. Moreover, given the inherent variability of traffic counts this approach is likely to lead to unfeasible solutions. As an alternative, the authors propose to integrate the traffic counts information by incorporating a second objective function that seeks to minimize the error in the estimation of traffic counts.

The TD-FTS formulation consists of a least squares function of traffic estimation error and an entropy maximization formulation of tour flows. The former is a function that minimizes the squared errors between observed and estimated traffic volumes for each time interval. The latter obtains the most probable meso states (i.e., time-dependent tour flows) comprised of micro states (i.e., time-dependent freight vehicle journeys), that are consistent with a set of macro states (i.e., trip-ends and total tours impedance). The consideration of these objectives reveals a tradeoff between the model's ability to replicate traffic vis-à-vis the weight given to the trip-ends and total cost in the network. On the one hand, traffic counts are reasonably accurate; though are highly variable, are influenced by seasonality, and are only available for small portions of the network. On the other hand, trip-ends could be reasonably well estimated for entire urban areas; though estimating the total cost in the network could be challenging. As a result, the analyst in charge must decide what is the "best" balance between these objectives as this person is the one best positioned to know the strengths and weakness of the data used.

The relationship between macro states, meso states and micro states is of great importance to the model. The first macro state is a TAZ's total freight trip-ends, which corresponds to the number of trips attracted or/and produced in a TAZ. To comply with this macro state, the summation of freight vehicles on tours that visit the TAZ should be equal to the trip-ends of this TAZ, where TAZs' trip-ends are specific to an industry sector and a time interval. The meso state is defined as the time-dependent flow on a tour serving a given industry sector. Trip-ends constraints can be considered in daily terms or per time interval.

In addition to satisfying the trip-ends constraints, the flows must meet overall total cost on the network. This is ensured by the impedance constraint that establishes that the summation of the costs assumed by individual vehicles following every tour is equal to an estimate of total cost for the network (or alternatively the total cost for each industry sector). As explained by Wilson (1970), this constraint ensures that feasible meso states generate a pattern of flows with a mean unit travel cost consistent with reality. In this model, the impedance of a tour is the summation of the travel time and the service time. The travel time corresponds to the time spent on the vehicle to reach the TAZs visited in the tour (which varies by time interval); the service time includes goods/equipment load and unload, handling time, and other activities that take place when the vehicle is parked for freight handling purposes.

The resulting model can be expressed as a mathematical program as follows:

PROGRAM TD-FTS-1

$$
\begin{gathered}
\underset{x_{m, y}^{d}}{\operatorname{Minimize}} \quad z_{1}(\mathbf{x})=\sum_{a=1}^{A} \sum_{k=1}^{K}\left(v_{a}^{k}-\sum_{d=1}^{K} \sum_{m=1}^{M} \sum_{y=1}^{Y} p_{a m}^{k d} \delta_{a m}^{k d} x_{m, y}^{d}\right)^{2} \\
\underset{x_{m, y}^{d}}{\operatorname{Maximize}} \quad W(\mathbf{x})=C_{X}^{x_{m, y}^{d}} \cdot C_{\left(X-x_{m, y}^{d}\right)}^{x_{m, y}^{d}}=\frac{X !}{\prod_{m, d, y}^{M, K} \chi_{m, y}^{d} !} \\
\text { Subject to }: \sum_{d=1}^{K} \sum_{m=1}^{M} \gamma_{j m}^{k d} x_{m, y}^{d}=D_{j, y}^{K} \quad\left(\lambda_{j, y}^{k}\right) \\
\sum_{d=1}^{K} \sum_{m=1}^{M} \sum_{y=1}^{Y} c_{m}^{d} x_{m, y}^{d}=C \quad(\beta) \\
x_{m, y}^{d} \geqslant 0
\end{gathered}
$$


where the variables/parameters are defined as:

$z_{1}(\mathbf{x}) \quad:$ Traffic replication function

$x_{m, y}^{d} \quad$ : Tour flow following tour $m$ starting at time interval $d$ and serving industry $y$

$\mathbf{x} \quad$ : Vector where each component is a flow $x_{m, y}^{d}$

$v_{a}^{k} \quad$ : Traffic volume at link $a$ during time interval $k$

$p_{a m}^{k d} \quad$ : The proportion of flow of tour $m$ starting at time interval $d$ traversing link $a$ during time interval $k$

$\delta_{a m}^{k d} \quad$ : Binary parameter equal to 1 if tour $m$ starting at time interval $d$ reaches link $a$ during interval $k$, otherwise zero

$W(\mathbf{x})$ : Entropy function

$C_{X}^{x_{m, y}^{d}} \quad$ : Combination of selecting $x_{m, y}^{d}$ from $X$

$X \quad$ : The total number of vehicle journeys in the network, $X=\sum x_{m, y}^{d}$ (includes multiple journeys done by the same vehicle during the day)

d : : Tour m's start time

$K \quad$ : Number of time intervals

$M \quad$ : Total number of tour sequences considered

$Y \quad$ : Total number of industry sectors considered

$\gamma_{j m}^{k d} \quad$ : Binary parameter equal to 1 if tour $m$ starting at time interval $d$ visits TAZ $j$ during interval $k$, otherwise zero

$D_{j . y}^{k} \quad$ : Trip demand of industry $y$ in TAZ $j$ at time interval $k$

$c_{m}^{d} \quad$ : Impedance of tour $m$ departing at time interval $d$

C : Total impedance in the network

$\lambda_{j, y}^{k} \quad$ : Lagrange multiplier associated with the demand on TAZ $j$ at time interval $k$ for industry $y$ constraint

$\beta \quad$ : Lagrange multiplier associated with total impedance constraint

The objective function described in Eq. (4) represents the number of ways in which the flows $x_{m, y}^{d}$ can be selected from $X$. Maximizing this expression is equivalent to maximize the entropy of a probability distribution in information theory, which allows to obtain the most probable flows (Wilson, 1970). This function includes a number of factorial terms that add notorious complexity to the optimization problem. Following Wilson (1970), the same result will be obtained if any monotonic function of $W$ is maximized. Thus, this equation can be simplified by taking natural logarithms to obtain:

$$
\underset{x_{m, y}^{d}}{\operatorname{Maximize}} z_{2}^{\prime}(\mathbf{x})=\ln (W(\mathbf{x}))=\ln (X !)-\sum_{d=1}^{K} \sum_{m=1}^{M} \sum_{y=1}^{Y} \ln \left(x_{m, y}^{d} !\right)
$$

The term $\ln (X !)$ can be dropped from the objective function assuming that the total number of vehicle journeys in the network is constant. Moreover, since maximizing $z_{2}^{\prime}(\mathbf{x})=\ln (W(\mathbf{x}))$ is equivalent to minimizing $z_{2}^{\prime \prime}(\mathbf{x})=-\ln (W(\mathbf{x}))$, it is possible to obtain the following objective function:

$$
\underset{x_{m, y}^{d}}{\operatorname{Minimize}} z_{2}^{\prime \prime}(\mathbf{x})=\sum_{d=1}^{K} \sum_{m=1}^{M} \sum_{y=1}^{Y} \ln \left(x_{m, y}^{d} !\right)
$$

To further simplify the formulation, most entropy based optimization models use the Stirling approximation to obtain a continuous and differentiable objective function. However, as opposed to OD trip distribution, the TD-FTS model is likely to produce low or even null flows for which Stirling approximation produces large errors. See de Grange and González (2012) for a discussion about the difficulties of using the Stirling approximation for low flows and the advantages of using Burnside approximation (Burnside, 1917). Replacing the factorial terms leads to an entropy function that is differentiable and continuous, and that is more amenable for computational optimization. Using the latter, the objective function can be modified by replacing the terms $\ln (x !)$ as follows:

$$
\ln (x !) \approx \frac{1}{2} \ln (2 \pi)+\left(x+\frac{1}{2}\right) * \ln \left(x+\frac{1}{2}\right)-\left(x+\frac{1}{2}\right)
$$

Thus, the mathematical program becomes:

\section{PROGRAM TD-FTS-2}

$$
\underset{x_{m, y}^{d}}{\operatorname{Minimize}} \quad z_{1}(\mathbf{x})=\sum_{a=1}^{A} \sum_{k=1}^{K}\left(v_{a}^{k}-\sum_{d=1}^{K} \sum_{m=1}^{M} \sum_{y=1}^{Y} p_{a m}^{k d} \delta_{a m}^{k d} x_{m, y}^{d}\right)^{2}
$$




$$
\begin{gathered}
\underset{x_{m, y}^{d}}{\operatorname{Minimize}} z_{2}(\mathbf{x})=\sum_{d=1}^{K} \sum_{m=1}^{M} \sum_{y=1}^{Y}\left(\left(x_{m, y}^{d}+\frac{1}{2}\right)\left(\ln \left(x_{m, y}^{d}+\frac{1}{2}\right)-1\right)\right) \\
\text { Subject to }: \sum_{d=1}^{K} \sum_{m=1}^{M} \gamma_{j m}^{k d} x_{m, y}^{d}=D_{j, y}^{k} \\
\sum_{d=1}^{K} \sum_{m=1}^{M} \sum_{y=1}^{Y} c_{m}^{d} x_{m, y}^{d}=C \\
x_{m, y}^{d} \geqslant 0
\end{gathered}
$$

In the TD-FTS model, each tour is defined by a home-base, the TAZs visited, and the tour starting time. As shown in Eqs. (11) and (13) of TD-FTS-2, the incidence matrices $\delta$ and $\gamma$ are crucial because they map the tour sequences to network links and they indicate which TAZs are visited by each tour, respectively. These matrices allow the model to consider the time-dependent effects on: the tour starting time; and the arrival to each TAZ and link under study. The time domain is discretized in intervals of $\Delta$ time duration and the arrival time to each TAZ and link is estimated using the tour sequences, the topology of the network, and the average travel and service times. For each tour $m$ starting at $d$, the binary variable $\delta_{a m}^{k d}$ indicates if tour $m$ reaches link a during the interval $k$, as shown in the following equation (the same equation is used for $\gamma_{j m}^{k d}$, but instead of marking when link $a$ is reached, the variable indicates when the tour reaches $\operatorname{TAZ} j$ ):

$$
\delta_{a m}^{k d}= \begin{cases}1, & \text { if }(k-1) \Delta \leqslant(d-1) * \Delta+\eta_{m}^{a}(d)<k \Delta \text { and } d \leqslant k \\ 0, & \text { otherwise }\end{cases}
$$

where:

$\eta_{m}^{a}(d) \quad$ The elapsed total time of tour $m$ that started at interval $d$ when it arrives to link $a$ (this includes service time in the previously visited centroids).

In essence, the time-dependent link-tour incidence matrix $(\delta)$ define the time interval within which a tour reaches each link; and therefore, when multiplied by the traffic assignment proportion $(p)$, determines which combinations of tours meet trip-ends constraints and closely reproduce the time-dependent traffic counts.

The tour flows determine the OD flows, as follows:

$$
x_{i j}=\sum_{d=1}^{K} \sum_{m=1}^{M} \psi_{i j m} x_{m}^{d} \quad \forall i, j \in\{1,2, \ldots, N\}
$$

where

$$
\begin{array}{ll}
x_{i j} & \text { Number of vehicles traveling from } \operatorname{TAZ} i \text { to TAZ } j, \\
\psi_{i j m} & \text { A binary variable equal to } 1 \text { if OD pair } i j \text { is in tour } m \text {, otherwise } 0 .
\end{array}
$$

As a tour sequence is defined by the TAZs visited, each pair of trip-ends is equivalent to an OD pair. Therefore, it is possible to convert the traffic assignment proportion from OD pairs to tours, as follows:

$$
p_{a m}^{k d}=\sum_{i j} \psi_{i j m} p_{a, i j}^{k d}
$$

There are obvious interconnections between tour flows and impedances, and the OD matrices and the corresponding impedances. The impedance of each tour can be estimated as the summation of the impedances of the OD pairs belonging to the tour including service times:

$$
c_{m}=\sum_{i j} \psi_{i j m} c_{i j}+\sum_{j} \gamma_{j m} c_{j}^{\prime}
$$

where

$$
\begin{array}{ll}
c_{i j} & \text { Impedance between TAZs } i \text { and } j, \\
c_{j}^{\prime} & \text { Service time at TAZ } j .
\end{array}
$$

It is important to note that the TD-FTS includes the service time in the tour impedances, while other applications only consider the impedance between OD pairs and neglect service times. Furthermore, Eq. (17) shows that the OD flows are a consequence of tour flows, which in turn depend on the tour impedances instead of the impedances between the OD pairs. These are important observations as they show the fallacy of modeling urban freight using trip-based approaches that implicitly assume that the freight trips-part of a tour-are independent of each other. The models presented in this paper are, thus, expected to enhance current modeling techniques because the unit of flow (i.e., tours) is consistent with actual freight vehicle movements. 
An important aspect that deserves discussion is how to reconcile the different objectives of the formulation developed, i.e., Program TD-FTS-2, which considers a metric of likelihood of the tour flows (maximum of entropy) and the ability of the tour flows to replicate the observed traffic counts (minimum of squared errors). From the standpoint of a decision maker (DM) that has to use the proposed model, balancing these (conflicting) objectives resembles a multicriteria decision process in which the DM has to decide on the preferred solution given his preference structure (which represents his intrinsic valuations concerning the multiple criteria being considered). From the vantage point of decision theory, which is the perspective adopted in this paper, the approaches used in the OR literature are lacking. Section 4 proposes the Multi-attribute Value (MAV) function approach as the most appropriate technique to combine the two objectives into a single objective.

\section{Multi-attribute value formulation}

The first part of this section presents the mathematical considerations to combine multiple objectives, while the second part uses the MAV theory to obtain a program with a single objective function from Program TD-FTS-2.

\subsection{Mathematical considerations for multiple objectives}

Generally speaking, decision-making implicitly requires the use of a preference order to rank the available alternatives, and select the most appropriate one (Chankong and Haimes, 1983). In problems where there is only one criterion, such as cost minimization, the preference order follows the natural order of real numbers. In these cases, the application of optimization techniques leads directly to the best choice. However, there are other problems with multiple objectives where the preference structure is not so clear. In these cases, utility theory can be used to derive the underlying preference structure, and in the absence of uncertainty, the alternative with the highest utility will represent the best choice.

Measuring the preferences and characterizing the underlying preference structure of decision-making problems requires investigating the properties of the preferred order to select an appropriate scale of measurement. In general, to guarantee the existence of a real-valued function, the preference relationship $(R)$ between alternatives $x_{a}, x_{b}$ and $x_{c}$, should be transitive (i.e., $x_{a} R \mathrm{x}_{b}$ and $x_{b} R x_{c}$ imply $x_{a} R x_{c}$ ), reflexive, i.e., $x_{a} R x_{a}$, and connected (i.e., $x_{a} R x_{b}$ and/or $x_{b} R x_{a}$ for all $x_{a} \in X$ and $x_{b} \in X$, where $X$ is the set of all feasible alternatives). The set of alternatives can be partitioned into a countable number of indifference classes (i.e., for $x_{a}$ and $x_{b}$ in the same class $x_{a} \succcurlyeq x_{b}$ and $x_{b} \preccurlyeq x_{a}$, or equivalently $x_{a} \sim x_{b}$ ). As these classes are countable, there exists a real-valued function called the value function (i.e., $v_{i}$ ), such that:

$$
x_{a} \succcurlyeq x_{b} \text { if and only if } v\left(x_{a}\right) \geq v\left(x_{b}\right)
$$

These premises are also applicable to the multi-objective case, where the value functions $\left(v_{i}\right)$ can be estimated for each attribute, and then combined into a single utility function. The general form of the resulting Multi-attribute Value (MAV) function can be stated as follows:

$$
U(\mathbf{x})=f\left[v_{1}\left(z_{1}(\mathbf{x})\right), \ldots, v_{n}\left(z_{n}(\mathbf{x})\right)\right]
$$

where $\mathbf{x}$ is a particular alternative, $z_{i}(\mathbf{x})$ is the consequence of this alternative in terms of attribute $i, v_{i}$ designates the value function for attribute $i$, and $f$ is a function that relates the individual values to the overall utility $U$. In some cases, additivity properties can be assumed for the value functions, and the function $f$ can be represented by a convex combination of the value functions, resulting in the following equation:

$$
U(\mathbf{x})=\alpha_{1} v_{1}\left(z_{1}(\mathbf{x})\right)+\cdots+\alpha_{n} v_{n}\left(z_{n}(\mathbf{x})\right)
$$

where $\alpha_{i}$ is a scale factor, such that $\alpha_{i}>0$ and $\sum_{i-1}^{n} \alpha_{i}=1$. The scale factors, as well as the component value functions, are estimated through an in-depth interview process with the DM.

Variations of this analytical framework can support decision-making under uncertainty. In essence, the decision theory adopted in this paper is based on the premises that a DM preference can be quantified, measured and represented in the form of a real-valued function, referred to as the MAV function. As explained by Chankong and Haimes (1983), this approach can be implemented in five steps: (i) verify the existence of a MAV function, (ii) select a suitable form for the function, (iii) construct appropriate component functions, (iv) determine scaling constants, and (v) check consistency and perform final analysis. It is informative to use MAVs theory to analyze three of the most common approaches used to construct a single objective function from a set of individual objective functions: convex combination, normalization, and the Pareto frontier.

The convex combination approach relies on the use of $n$ parameters $\theta_{i}$, such that $\sum_{i=1}^{n} \theta_{i}=1$, to construct a composite value function directly from the original ones, as shown below:

$$
Z=\theta_{1} z_{1}(\mathbf{x})+\cdots+\theta_{n} z_{n}(\mathbf{x})
$$

It is important to highlight the differences between Eq. (22), which is the simplest case for the MAV, and Eq. (23). While in Eq. (22) the composite function is a convex combination of individual value functions, in Eq. (23) the composite function is a convex combination of the observable consequences of each alternative. There are a number of problems with the latter. First, there is the issue of compatibility of units which arises frequently because in most multi-objective formulations the component objective functions represent different aspects of the phenomenon being modeled. A second 
issue is that the convex combination approach implicitly assumes linear valuations, and constant marginal rates of substitution among the objectives. As a result, decision problems where satiation is found, or nonlinear valuations, cannot be properly modeled.

The normalization approach, often applied to control problems, is based on Bryson's rule (Franklin et al., 1997). This approach scales the variables in the objective function by multiplying the outcome of each attribute $z_{i}(\mathbf{x})$ by the inverse of the attribute maximum expected magnitude, i.e., $\frac{1}{\max z_{i}(\mathbf{x})}$. The unit-free composite function becomes:

$$
Z=\frac{z_{1}(\mathbf{x})}{\max z_{1}}+\frac{z_{2}(\mathbf{x})}{\max z_{2}}
$$

This technique resolves the issue of units, though, is unable to overcome the limitation concerning valuation because the contributions to $Z$ from each normalized function are not related to the value they represent to the DM. In essence, this technique applies scale factors that convert each function into proportions of the maximum expected magnitude; but, by assigning the same value to each attribute, completely disregards the valuation problem.

The Pareto efficient frontier studies the space of consequences for the different alternatives, and identifies the non-dominated alternatives. Alternative $x_{a}$ is said to dominate an alternative $x_{b}$, if the consequences of $x_{a}$ are at least as good as the ones for $x_{b}$ for every attribute, and strictly better for at least one (Keeney and Raiffa, 1993). Mathematically, if the consequences for alternative $x_{a}, z_{1}\left(x_{a}\right), \ldots, z_{n}\left(x_{a}\right)$, and the ones for alternative $x_{b}, z_{1}\left(x_{b}\right), \ldots, z_{n}\left(x_{b}\right)$, follow:

$$
z_{i}\left(x_{a}\right) \geqslant z_{i}\left(x_{b}\right), \quad \forall i \text {, and } z_{i}\left(x_{a}\right)>z_{i}\left(x_{b}\right), \text { for some } i .
$$

Then, alternative $x_{a}$ dominates alternative $x_{b}$. This idea of dominance gives preponderance to the ordinal (i.e., $z_{i}\left(x_{a}\right)>z_{i}\left(x_{a}\right)$ ) nature of the consequences over their cardinal nature (i.e., $\left.z_{i}\left(x_{a}\right)-z_{i}\left(x_{b}\right)>z_{j}\left(x_{a}\right)-z_{j}\left(x_{b}\right)\right)$. In essence, Pareto efficient frontiers can be used to eliminate all dominated alternatives, so that the DM can select the solution that best fits his preferences. The main limitation of the Pareto frontier is that the ordering relation in the Euclidean space is only a partial order and does not hold, necessarily, a relationship with the preference relation which involves subjectivity and value judgment. To overcome the valuation problem, each point in the frontier can be represented by a convex combination $\sum_{i=1}^{n} \lambda_{i} z_{i}(\mathbf{x})$ where $\sum_{i=1}^{n} \lambda_{i}=1$, and the DM can choose the optimal alternative based on his preferred marginal rate of substitution among attributes. However, implementing this solution takes the problem back to the problem of convex combinations discussed before, where valuation is assumed to be linear and proportional to the magnitude of the attribute. The Pareto frontier is a powerful technique that can be used as a pre-screening process, but requires a complementary methodology that formalizes the preference structure and incorporates it in the optimization process.

The review presented in this section reveals some of the issues associated with the techniques commonly used to reconcile different objectives in optimization problems. The fundamental problem with convex combinations, normalization and Pareto frontiers is that they do not treat scaling and valuation as two different issues, they are unable to deal with nonlinear valuations, and they do not formalize the preference structure of the DM. The MAV theory described in this section can be used to translate the Bi-Criteria FTS model presented in Section 3 (TD-FTS-2) into an optimization program with a single objective function.

\subsection{Incorporation of the MAV function}

Based on the MAV theory, Eq. (22) can be used to combine the two objective functions of TD-FTS-2 into a single utility function, which can then be maximized (or its negative minimized) as follows:

\section{PROGRAM TD-FTS-3}

$$
\begin{aligned}
& \underset{x_{m, y}^{d}}{\operatorname{Minimize}}-U\left(z_{1}(\mathbf{x}), z_{2}(\mathbf{x})\right)=-\alpha_{1} v_{1}\left(z_{1}(\mathbf{x})\right)-\alpha_{2} v_{2}\left(z_{2}(\mathbf{x})\right) \\
& \text { Subject to }: \sum_{d=1}^{K} \sum_{m=1}^{M} \gamma_{j m}^{k d} x_{m, y}^{d}=D_{j, y}^{k} \\
& \sum_{d=1}^{K} \sum_{m=1}^{M} \sum_{y=1}^{Y} c_{m}^{d} x_{m, y}^{d}=C \\
& x_{m, y}^{d} \geqslant 0 \text {, }
\end{aligned}
$$

The reformulated TD-FTS estimates the tour flows that better fit the preference structure of the DM. A particular case of this model is the Static FTS (S-FTS), in which only one time interval is considered. The first order and second order conditions of TD-FTS-3 were derived and are provided in the appendices. To complement the study of the first and second order conditions, the convexity of the model must be studied to envisage feasible value functions specifications. 


\subsection{Convexity}

As the constraints of TD-FTS-3 are linear, the objective function is being minimized over a convex set, and it suffices to prove that the objective function is convex to show that the TD-FTS-3 problem is convex. As shown in Eq. (26), the objective function of TD-FTS-3 depends on: the traffic replication function, the entropy function, the value functions and the scale factors. According to the principle of convexity in composition (Rockafellar and Wets, 1998), if both $z_{1}(\mathbf{x})$ and $z_{2}(\mathbf{x})$ are convex and $-\alpha_{1} v_{1}$ and $-\alpha_{2} v_{2}$ are non-decreasing, then the objective function $-U$ is convex. The convexity of $z_{1}(\mathbf{x})$ can be proved using again the principle of convexity in composition: as $z_{1}(\mathbf{x})$ is the 2-norm function of a linear function of $\mathbf{x}$, thus $z_{1}(\mathbf{x})$ is also convex, refer Rockafellar and Wets (1998). In the case of $z_{2}(\mathbf{x})$, the proof requires the derivation of the Hessian, as follows:

$$
\frac{\partial z_{2}^{2}(\mathbf{x})}{\partial x_{m 1, y 1}^{d 1} \partial x_{m 2, y 2}^{d 2}}=\left\{\begin{array}{cc}
\frac{1}{\chi_{m^{\prime} y}^{d}+\frac{1}{2}} & \text { for } m_{1}=m_{2}, d_{1}=d_{2}, y_{1}=y_{2} \\
0 & \text { otherwise }
\end{array} \quad \forall m \in M, d \in K, y \in Y\right.
$$

As shown in Eq. (30), the symmetric structure of the matrix and its nonnegative components reveal that the Hessian is strictly positive definite (strictly because at least one $x_{m^{\prime} y^{\prime}}^{d^{\prime}}$ will always be positive). Therefore, $z_{2}(\mathbf{x})$ is strictly convex. In terms of the $-\alpha_{1} v_{1}$ and $-\alpha_{2} v_{2}$, some restrictions apply to guaranty the convexity of TD-FTS-3. Table 1 summarizes the different cases for which the TD-FTS-3 problem is convex to shed light on the functional forms that can be used to model the DM preferences.

As shown, three different functional forms are proposed. Depending on the specification of the function, some conditions must be respected to have a convex function. The constraints imposed by the convexity condition 1 determine the sign of the function parameters $(a$ and $b$ ); for the linear and the logarithmic functions, the sign lines up with what would be expected for a rational DM (i.e., higher values of $z_{i}(\mathbf{x})$ decrease utility). The constraints imposed by convexity condition 2 on the quadratic function limit the functions to the range where they are non-increasing monotonic functions, which are basically the cases where value functions represent relationships that are transitive, reflexive and connected.

\section{Application of the TD-FTS-3 model}

The lack of data about urban freight and the absence of an appropriate framework for data collection are important obstacles for model development. If and when freight data are collected, they are too aggregated to analyze and characterize urban freight. Holguín-Veras and Jaller (2014) identified the following data as key to develop and calibrate urban freight demand models:

- Information into logistical patterns of flows: identifies the agents, their functions and their interactions on the system being modeled.

- Freight generation data: study the production and consumption of freight by studying the transactions between the shipper and the receiver of cargo.

- Freight trip generation data: estimate the number of freight trips needed to transport the freight generated by studying the logistic decisions of freight agents.

- Freight vehicle tours data: characterize the freight vehicles movements, by studying the sequence and location of customers (i.e., shipper and receivers) visited by freight vehicles, the origin and destination of commodity flows along the tour, and data about empty trips.

- Economic characteristics and spatial distribution of freight agents: used as attributes to characterize the behavior and patterns of freight agents, it includes business size, number and types of trucks, inventory policies, hours of operations, industry segment, and spatial location, among others.

- Network topology and traffic counts: describe the network characteristics, and provide the link between freight vehicle flows and the use of infrastructure.

- Other economic data: characterize detailed aspects of freight demand used to study production and demand functions of commodities in each region, used for spatial price equilibrium models and technical coefficients of Input-Output models.

Table 1

Value functions specifications for the MAV function.

\begin{tabular}{|c|c|c|c|c|}
\hline Case & Function & Specification & Condition 1 & Condition 2 \\
\hline 1 & Linear function $\left(v_{i}\right)$ & $b z_{i}(\mathbf{x})+c$ & $b<0$ & n.a. \\
\hline 2.a. & Quadratic function $\left(v_{i}\right)$ & $a z_{i}(\mathbf{x})^{2}+b z_{i}(\mathbf{x})+c$ & $a>0$ & $z_{i}(\mathbf{x}) \leqslant-\frac{b}{2 a}$ \\
\hline 2.b. & & & $a<0$ & $z_{i}(\mathbf{x}) \geqslant-\frac{b}{2 a}$ \\
\hline 3 & Logarithmic function $\left(v_{i}\right)$ & $a \ln \left(z_{i}(\mathbf{x})\right)$ & $a<0$ & n.a. \\
\hline
\end{tabular}


As shown, the data needs are quite comprehensive, which might be a challenge for most agencies to collect. In contrast, TD-FTS models require less data: freight trip generation data, spatial distribution of freight agents, network topology and traffic counts.

\subsection{Data requirements of the TD-FTS-3}

The TD-FTS-3 seeks to obtain the most likely tour flows, using data on freight flows that are available in a typical urban area or that can be estimated using complementary models. The main inputs for the model are the network topology, the traffic counts, the fraction of traffic flow traveling between TAZs using each link, the trip-ends for each TAZ, and the tour sequences. The first two inputs are available for most urban areas, though the passing-through freight vehicle have to be estimated from a regional/national level model (or data) and deducted from the traffic counts. However, the fraction of traffic flow traveling between each pair of TAZs using each link $\left(\bar{p}_{w}^{a}\right)$ requires a preliminary traffic assignment (based on an all or nothing or a multi-path algorithm), the trip-ends for each TAZ can be derived from observed tour flows if complete information on tour flows is available (which is rarely the case) or obtained through the application of freight trip generation models (FTG), and the tour sequences can be inferred from GPS data and/or generated through simulation or analytical techniques.

The use of establishment-based FTG models and freight vehicles tour sequence simulations is a crucial aspect of the TD-FTS because it provides the link between the economic activities performed by the various economic agents (i.e., shippers, carriers and receivers) and the resulting freight traffic. FTG models use statistical techniques to estimate the amount of freight trips attracted and produced at the establishment level for different industry sectors based on employment, area, and other variables related to land-use (Holguín-Veras et al., 2012; Lawson et al., 2012; Sanchez-Diaz et al., 2014). Quantifying FTG at the establishment level allows to include all type of vehicles (e.g., pickup, vans, light tuck, and heavy vehicles) and have a comprehensive picture of the freight vehicles in the network. Their main advantage is their solid behavioral foundations and their ability to study changes in traffic produced by new policy, new developments and other land-use changes. After aggregation, the output of FTG models corresponds to the trip-ends from freight vehicles tours used in the FTS.

The high penetration of GPS technologies and its increasing use for business efficiency has increased the availability of comprehensive GPS data in the last decade. Applying robust learning methods (e.g., support vector machine) to these data allows to differentiate between delivery and non-delivery stops, estimate service times, and derive tour sequences (Yang et al., 2014). However, since GPS only provide a partial view of freight activity, because numerous small carriers refuse to use GPS devices, they must be complemented with other data, such as travel inventories or with simulated tour sequences to avoid costly data collection efforts. Although high quality tour data will produce better results, complementing existing data with simulated tour sequences can provide good estimates for forecasting.

The tour sequences can be generated using one of the approaches described in the literature review. The selection of the technique depends on the data available. In the cases of limited data on commodity flows and/or production-consumption links, which is often the case for cities, the most appropriate approach is to simulate a set of feasible tour sequences using basic assumptions. The technique used in this paper is based on the behavioral simulation developed by Silas and Holguín-Veras (2009) and Holguín-Veras and Aros-Vera (2014) to assess the effectiveness of off-hour deliveries. Essentially, for each tour sequence: an industry sector is randomly selected, a tour length (i.e., number of TAZs to visit) is selected based on carriers data, the home base of the tour and the TAZs to be visited are selected (the probability of being selected is proportional to the contribution of the TAZs to the overall city's FTG), and a VRP is used to optimize each tour sequence. After generating the set of feasible tour sequences, the TD-FTS assigns flow to the most likely tour sequences ensuring that trip-ends constraints and the cost constraint are met.

\subsection{Application for forecasting purposes}

The models TD-FTS- 1 and TD-FTS-2 can be used to estimate a Pareto frontier but the DM would have to choose the solution among a large set of alternatives, which is inconvenient. Therefore, this section focuses on the application of the TD-FTS-3. As explained in Section 4, the TD-FTS-3 estimates the time-dependent freight tour flows that best match the DM preference structure, which is defined as a MAV function of the traffic count replication error and an entropy measure (related to the likelihood of the tours being the correct ones that match the trip-ends and total cost in the system). Although this approach works well to estimate the tour flows for a calibration year, for which traffic counts are known, it cannot be used for forecasting purposes because the traffic in the network is unknown. In order to deal with this challenge, the authors developed a FTS formulation (TD-FTS-4) that uses the results from TD-FTS-3 as the input to calibrate a reduced model. The authors' conjecture is that this reduced model, by building on the TD-FTS-3 results, will provide an approximation to TD-FTS-3 that will be suitable for forecasting purposes.

The objective function of TD-FTS-4 is formulated using a penalty method that ensures that the parameters reproduce the tour flows from TD-FTS-3 without depending on traffic counts, and obtains a set of Lagrange multipliers that have as unit the entropy of the system. Although the decision variable is the tour flows $\overline{x_{m, y}^{d}}$, the outputs of interest for the TD-FTS- 4 are the parameters $\overline{\lambda_{j y}^{k}}$ for each TAZ and time interval, and the parameter $\bar{\beta}$ for the cost constraint. The resulting formulation is: 


\section{PROGRAM TD-FTS-4}

$$
\begin{aligned}
& \text { Minimize } \quad z(\overline{\mathbf{x}}, \mathbf{x})=\sum_{d=1}^{K} \sum_{m=1}^{M} \sum_{y=1}^{Y}\left(\left(\overline{x_{m, y}^{d}}+\frac{1}{2}\right)\left(\ln \left(\overline{x_{m, y}^{d}}+\frac{1}{2}\right)-1\right)\right)+p\left(\overline{x_{m, y}^{d}}-x_{m, y}^{d}\right)^{2} \\
& \text { Subject to }: \sum_{d=1}^{K} \sum_{m=1}^{M} \gamma_{j m}^{k d} x_{m, y}^{d}=D_{j, y}^{k}\left(\overline{\lambda_{j, y}^{k}}\right) \\
& \sum_{d=1}^{K} \sum_{m=1}^{M} \sum_{y=1}^{Y} c_{m}^{d} x_{m, y}^{d}=C \quad(\bar{\beta}) \\
& x_{m, y}^{d} \geqslant 0, \quad \forall m \in M, d \in K, y \in Y
\end{aligned}
$$

where

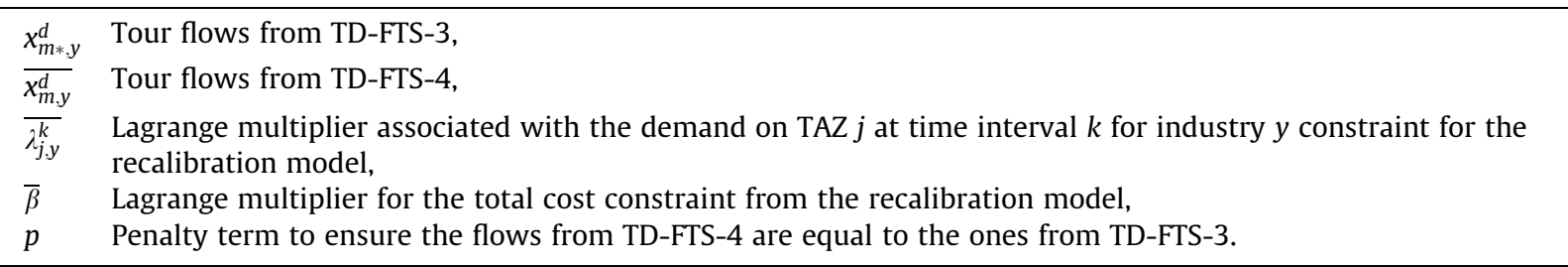

Similarly to the GM and the S-EM models described in the literature review, the result is a set of parameters for the trip-ends constraints and for the impedance constraint. For forecasting purposes, a new model referred to as TD-FTS-5 is introduced. This model can incorporate changes in land use, new policies, new developments (through forecasted FTG estimates), and changes in the infrastructure (through changes in the network topology), among others. The fundamental assumption of the TD-FTS-5 is that the impedance parameter remains constant for the forecasted scenario, resulting in a restricted model with the following form:

PROGRAM TD-FTS-5

$$
\begin{aligned}
& \underset{\overline{\overline{x_{m, y}^{d}}}}{\operatorname{Minimize}} z(\overline{\overline{\mathbf{x}}})=\sum_{d=1}^{K} \sum_{m=1}^{M} \sum_{y=1}^{Y}\left(\left(\overline{\overline{x_{m, y}^{d}}}+\frac{1}{2}\right)\left(\ln \left(\overline{\overline{x_{m, y}^{d}}}+\frac{1}{2}\right)-1\right)\right)+\bar{\beta}\left(\overline{\bar{C}}-\sum_{d=1}^{K^{\prime}} \sum_{m=1}^{M} \sum_{y=1}^{Y} \overline{\overline{c_{m}^{d} x_{m, y}^{d}}}\right) \\
& \text { Subject to }: \sum_{d=1}^{K} \sum_{m=1}^{M} \overline{\overline{\gamma_{j m}^{k d}}} \overline{\overline{\bar{x}_{m, y}^{d}}}=\overline{\overline{D_{j, y}^{k}}} \quad\left(\overline{\overline{\lambda_{j, y}^{k}}}\right) \\
& \overline{\overline{x_{m, y}^{d}}} \geqslant 0
\end{aligned}
$$

where

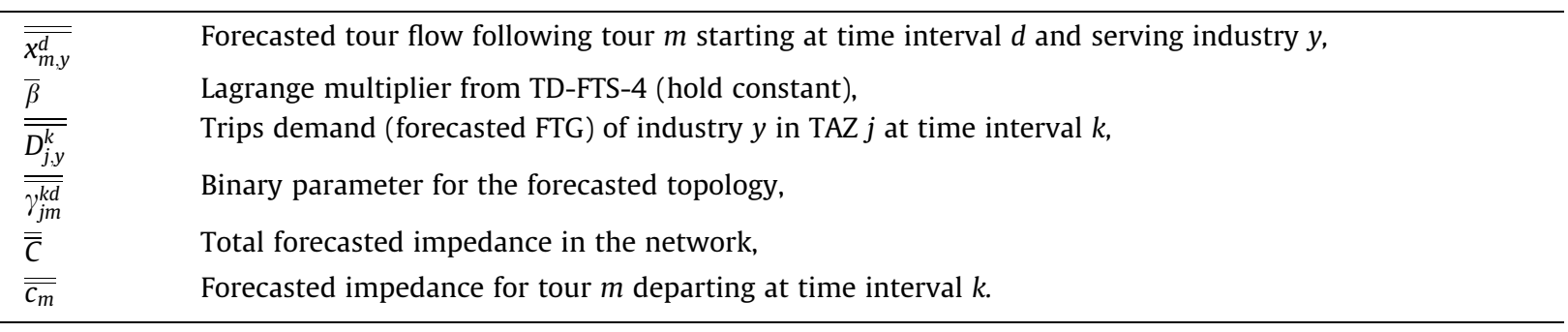

The TD-FTS- 5 model redistributes the flows among tour sequences to match the new FTG estimates while conserving the impedance parameter from the recalibration phase. Tour sequences that are improbable are expected to receive a null flow. It is important to note that, the tour sequences are prone to change because they represent operational decisions resulting from demand and from perceived network conditions. This highlights the importance of having a wide range of tour sequences as inputs: tour sequences that are assigned null flows in the calibration scenario may be assigned larger flows in the forecasting scenario as a result of changes in supply and demand.

\section{Case study: the Denver Region}

The Metropolitan Area of Denver (Colorado) was used to assess the effectiveness of the TD-FTS model. This section includes a description of the case study, followed by the results from the application of the TD-FTS models and a sensitivity analysis.

\subsection{Case study description}

The data were provided by the Denver Regional Council of Governments (DRCOG), which conducted surveys and collected GPS data from commercial vehicles in 1998 and 1999 as part of an overall Travel Behavior Inventory project (Denver 
Regional Council of Governments and Parsons Transportation Group Inc., 2001). It is worthy of mention that the designation of "commercial" vehicles include all vehicles used for commercial purposes, mostly for freight and service activities. The original data included information about industry sectors using the Standard Industry Classification (SIC) codes (Pearce, 1957), tour sequences, commercial vehicles tour flows, location and duration of stops, and trip purpose. The data were analyzed by Holguín-Veras and Patil (2005), who found that 344,000 trips are made by commercial vehicles in the region every day. This number includes multiple tours done by a single vehicle in one day. From these trips, about $13.12 \%$ have as purpose a service call, while $47.34 \%$ are freight related, and $24.33 \%$ have as purpose the return to the home base. In terms of industry sectors, the data shows that the service industry and public buildings generate the largest amount of trips, with $25 \%$ and $19 \%$. For the freight-intensive industries, retail is the one generating the largest traffic (16\%), followed by manufacturing ( $8 \%$ ), construction (8\%), wholesale (7\%), transportation (5\%), and mining and agriculture (about $1 \%$ each). The "other" sectors accounts for about $8 \%$ of the flows.

The authors decided to apply the models developed in the paper to the entire DRCOG data which, as said, includes both freight and service vehicles. Two reasons support this decision. First, although originally developed with freight applications in mind, the TD-FTS models can be applied to any form of transportation where tour-based behavior is significant. Second, using the entire dataset provides a better assessment of the model's performance because more data are being used. To apply the model to the Denver data, the commercial vehicles tour sequences were geo-located and aggregated using the zoning system from the Colorado Department of Transportation. The zoning system was aggregated to ensure that all TAZs were visited by a meaningful number of commercial vehicles.

As discussed before, the time-dependent nature of the problem requires the specification of tour departure times, the travel times on the network, and traffic volumes by time of day. Therefore, a set of traffic counts per time interval provided by the DRCOG and the historical series of travel time provided by the American Transportation Research Institute (ATRI) (Jones et al., 2005) were used to estimate travel times. The day was discretized in 6 non-overlapping 4-h time intervals, defined as follows: AM 1 from 12:00 am to 3:59 am, AM 2 from 4:00 am to 7:59 am, AM 3 from 8:00 am to 11:59 am, PM 1 from 12:00 pm to 3:59 pm, PM 2 from 4:00 pm to 7:59 pm, and PM 3 from 6:00 pm to 11:59 pm. The time distribution of the traffic counts in the home base county of the tour were used as a rough approximation of the flow distribution among tours with the same structure but different departure times. The analysis of the GPS data revealed that only five out of the eight counties forming the region served as home base of tour sequences (Denver, Adams, Douglas, Jefferson and Boulder). The traffic proportions for these counties were on average 3\%, 18\%, 24\%, 28\%, 21\% and 6\% for AM1, AM 2, AM 3, PM 1, PM 2, and PM 3 intervals, respectively. In terms of travel times, according to the historical data provided by ATRI, the commercial vehicles speeds in the Denver Region vary between $18.23 \mathrm{miles} / \mathrm{h}$ and $71.36 \mathrm{miles} / \mathrm{h}$ depending on the street, the proximity to the Central Business District, and time of the day. The service time at each TAZ, estimated from the DRCOG GPS data, ranged from 10 to $92 \mathrm{~min}$, with an average of $29.9 \mathrm{~min}$ per TAZ and a median of $23 \mathrm{~min}$ per TAZ. The post-process and the aggregation of the data resulted in 483 TAZs with an average demand for commercial vehicles of 439 trips/day supplied by 502 tour sequences that became 3012 in the time-dependent case, and 6024 when the daily tours are divided into the 12 industry sectors considered. The average number of TAZ visited per tour is 3.6 and the overall cost on the network for the case study was estimated to be approximately $139,461 \mathrm{~h}$ /day.

Although in reality the TAZs are connected by a set of individual link segments, for simplicity, the TAZs were assumed to be connected by super-links that encompass multiple link segments. In this case, the super links represent the shortest path between the centroids, thus the flow on super links is equivalent to the flow on paths. Moreover, since the purpose of this paper is to assess how well the TD-FTS reproduces tour flows, the observed traffic volumes were not collected directly from the field but instead the actual tour flows were assigned to the super links. This procedure allows to assess the performance of the model, isolating it from other sources of error as the ones associated with FTG estimates, traffic counts, and passing-through flows. According to previous research done by the authors (Sanchez-Diaz, 2014), it is not necessary to use all the super-links to apply the FTS, so only the 50\% of the super-links with the highest flows were used for the case study.

\subsection{Application of the TD-FTS-2}

This section describes the results from applying the TD-FTS-2 to the case study. The output of the model is a Pareto Frontier where each point represents a feasible solution for the synthesis problem.

To simulate the effects of imperfect information about tour sequences, the authors increased the number of tour sequences used by the models beyond the tour sequences captured in the DRCOG data. To this effect, 251 new sequences (50\% of the original number) were generated using the Behavioral Micro-Simulation (Silas and Holguín-Veras, 2009; Holguín-Veras and Aros-Vera, 2014). This technique conducts a simulation of the tours using trip-ends estimated, and the number of stops per tour. The consideration of additional tour sequences provides a more challenging environment to assess the models' performance.

After setting up the case study, the authors applied the TD-FTS-2 to obtain the Pareto frontier using the $\varepsilon$-constraint method (Chankong and Haimes, 1983). The application of this method results in a set of problems with traffic replication as the objective function while the entropy objective function becomes a constraint bounded by the aspiration level $\varepsilon$. Fig. 1 illustrates the results for the range of feasible entropy values. 


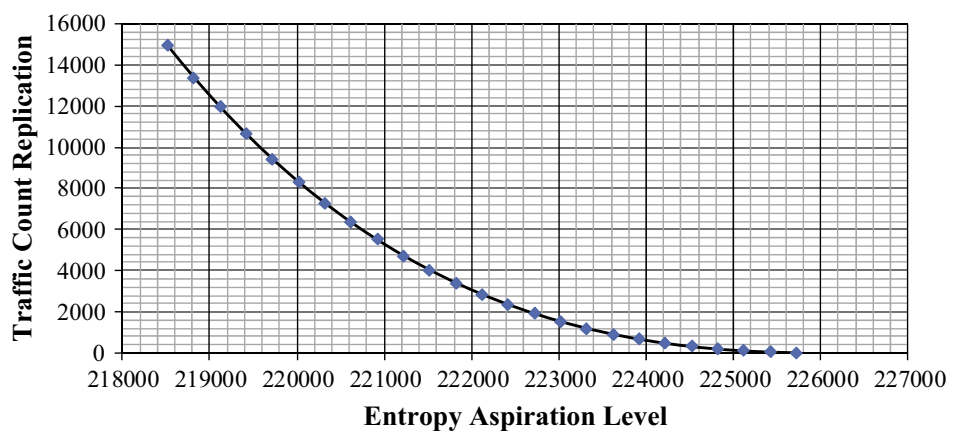

Fig. 1. Pareto efficient frontier.

As shown, the objective functions conflict with each other, though the tradeoff stabilizes for entropy values larger than 225,000 . For an entropy value of 219,000 , to decrease by 1 unit the traffic replication objective it is necessary to give up 5 units of entropy; while for 225,000 the traffic replication tend to be constant tending to 0 independently from the entropy. Another observation is the difference in the magnitude of the objectives; while entropy objective ranges between 218,500 and 225,000, the traffic replication objective ranges between 0 and 16,000.

Prior to estimating a convex function to explore the Pareto frontier, it is necessary to find an interpretation for each objective function, so that the DM can assign tradeoffs and select his best choice. The authors identified two basic criteria that the DM takes into account to evaluate the performance of the TD-FTS: the performance of the model in reproducing tour flows, and the sensitivity to the variation in traffic counts. These two criteria are closely related to the objective functions of the TD-FTS. It is expected that relaxing (increasing) the aspiration level for entropy leads to flows that reproduce traffic counts closely, which is appropriate when traffic counts are very accurate; while setting a tight (decreasing) aspiration level contributes to decrease the model dependence on traffic counts, and therefore to stabilize it in the presence of traffic counts with large variation.

To explore the relationship between the objective functions, and the ability to reproduce tour flows and the sensitivity to the variation of traffic counts, the authors simulated stochastic traffic counts that reproduce historical traffic volumes. The lognormal distribution was found to produce the best fit for the data in most cases (using a Smirnov-Kolmogorov test for each link and time interval), and the standard deviation parameter found ranged from $10 \%$ to $130 \%$ of the mean depending on the links. Thus, the traffic counts were assumed to follow lognormal distributions $\left(\mu, \sigma_{i}\right)$, where $\mu$ is the observed traffic counts and the standard deviation, $\sigma_{i}$, ranged from $0.10 * \mu$ to $1.30 * \mu$ for the different scenarios. For each of these standard deviation scenarios, the authors applied the TD-FTS-2 for each non-dominated point in the Pareto frontier of Fig. 1, and assessed its performance using the Mean Average Percentage Error (MAPE), defined in the following expression:

$$
M A P E=\sum_{d=1}^{K} \sum_{m=1}^{M}\left(\frac{a b s\left(x_{m}^{d, o b s}-x_{m}^{d, e s t}\right)}{x_{m}^{d, o b s}}\right)
$$

where

$$
\begin{array}{ll}
x_{m}^{d, o b s} & \text { Observed tour flow following tour } m \text { starting at time interval } d, \\
x_{m}^{d, \text { est }} & \text { Estimated tour flow following tour } m \text { starting at time interval } d .
\end{array}
$$

The outcome from this exploratory exercise is a detailed set of figures that illustrate the effect of entropy on the MAPE depending on the traffic counts distribution. Fig. 2 shows a 3-dimensional illustration of this relationship.

As shown in Fig. 2, for CV lower than 70\%, the lowest MAPE is achieved when the entropy constraint is relaxed, while for CV higher than 70\% the opposite is true. Another finding is that the slope of the curve representing MAPE vs. CV is significantly higher for relaxed entropy than for tight entropy. In other words, an increase in the standard deviation of traffic counts has larger effects on the MAPE when the entropy constraint is relaxed. Using these results, the DM can now select between non-dominated points in the Pareto frontier taking into account the expected MAPE and its expected standard deviation. Although the choice of the DM will define the best flow alternative, it does not provide a formal structure for his preferences or a set of parameters that could be used for forecasting. To this effect, it is necessary to estimate a MAV function that captures the DM preferences and use them to fuse the two objectives function into a single utility function.

\subsubsection{Estimation of the component value functions using the MAV}

Based on the application of the TD-FTS-2, it is possible to establish a function that relates the entropy and the traffic replication to an expected MAPE and an expected standard deviation of the MAPE, so that the DM choice becomes basically one between average performance and variance of the performance. A questionnaire was prepared by the authors, and used to inquire two DMs about their preferences and their rates of substitution between the two attributes. The DMs inquired are transportation engineers familiar with statistics concepts. Based on the responses to the questionnaires, the mid-value 


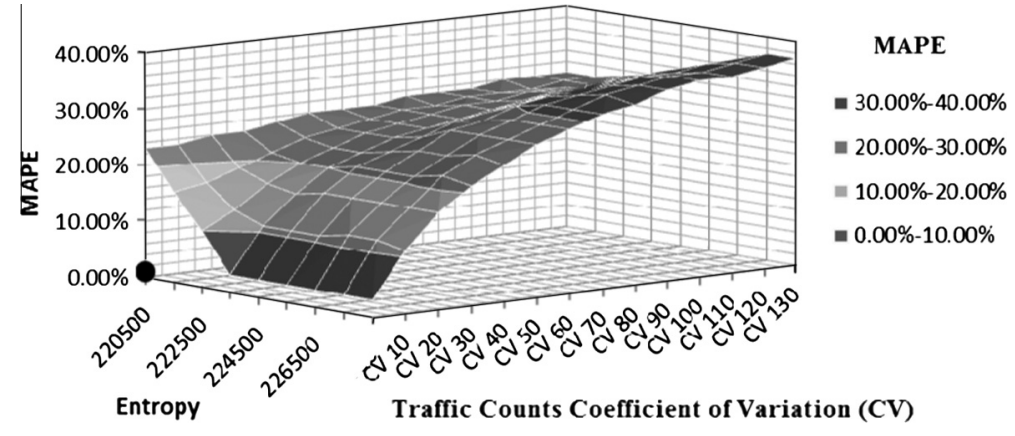

Fig. 2. MAPE as a function of entropy for different levels of traffic counts variation. Notes: MAPE: mean average percentage error, $\mathrm{CV}$ : coefficient of variation $(\sigma / \mu)$. Each point (CV, Entropy, MAPE) is obtained as the average for 1000 runs of TD-FTS-2. $\bullet$ : marks the origin.

splitting technique was used to estimate the value functions $v_{1}$ and $v_{2}$ for the median MAPE and its standard deviation, respectively, and obtain the utility function $U$, following (Keeney and Raiffa, 1993). The MAV functions result in the following utility functions for the two DMs:

$$
\begin{aligned}
& U_{A}\left(z_{1}(\mathbf{x}), z_{2}(\mathbf{x})\right)=0.54\left(18.6-4.19 .10^{-06} z_{1}(\mathbf{x})\right)+0.46\left(29.2-1.29 .10^{-04} z_{2}(\mathbf{x})\right) \\
& U_{B}\left(z_{1}(\mathbf{x}), z_{2}(\mathbf{x})\right)=0.78\left(15.75-1.22 * \ln \left(z_{1}(\mathbf{x})\right)\right)+0.22\left(29.7-1.32 .10^{-04} z_{2}(\mathbf{x})\right)
\end{aligned}
$$

where $z_{1}(\mathbf{x})$ is traffic replication function, $z_{2}(\mathbf{x})$ is the entropy function.

As shown, for DM A the scale factors are similar for both value functions, with a slight preference for traffic replication. Both value functions have negative linear coefficients, meaning that an increase in one of the objectives produce a decrease in utility. However, utility decreases faster when the entropy objective $\left(z_{2}(\mathbf{x})\right)$ increases than when traffic replication $\left(z_{1}(\mathbf{x})\right)$ increases. For DM B, the value functions are nonlinear and the scale factor is larger for the traffic replication value function, which reveals his inclination to reproduce traffic counts. For DM B's value functions the marginal utility decreases when the traffic replication objective increases. In addition to being conceptually valid, these coefficients must respect the conditions set in Table 1 to have a convex problem. These MAV functions are incorporated into TD-FTS-3, the model is calibrated and recalibrated (using TD-FTS-4) for the case study.

\subsection{Application of TD-FTS-3 and TD-FTS-4}

This section presents the results from applying the TD-FTS-3 which is constructed based on the objective functions from Eqs. (39) and (40), and the results from applying the recalibration formulation introduced as TD-FTS-4. The models are referred as S-FTS when only one time interval (static case) is considered. TD-FTS-3-A is the model for DM A, and TD-FTS-3-B is the model for DM B. To study each feature of the model thoroughly, the models assume a unique industry sector in the cases where industries are not relevant for the results. Two other entropy-based models are estimated to assess the TD-FTS performance through a comparative analysis: The traditional Gravity Model (GM) (Wilson, 1967), which imposes OD pairs structure to the flows; and the Static Entropy Maximization (S-EM) model (Wang and Holguín-Veras, 2009), which imposes a tour structure but does not incorporate information from traffic counts.

All the models were calibrated using the PATHNLP solver in GAMS (Brooke and Rosenthal, 2003), in a computer with an Intel Core Processor i- 5 with clockspeed $2.3 \mathrm{GHz}$ and $8.00 \mathrm{~GB}$ RAM. The DCGM took $0.56 \mathrm{~s}$ to complete the computations, the S-EM took $0.61 \mathrm{~s}$ and the TD-EM $92.82 \mathrm{~s}$, the S-FTS-3-A and S-FTS-3-B took 0.98 and $1.20 \mathrm{~s}$ respectively, and the TD-FTS-3-A and TD-FTS-3-B took 142.05 and $251.25 \mathrm{~s}$ respectively. The MAPE is used as the metric to compare the performance of the models. The analysis measures the ability to reproduce daily flows (in commercial trips) as well as the time-dependence of these flows. In the case of the GM the time-dependent flows were estimated in proportions that resemble the observed time of day split (2\%, $12 \%, 26 \%, 28 \%, 25 \%$ and $7 \%)$; while for the TD-EM and the TD-FTS-3-A/B models, the TD flows are a direct output of the model. The top of Table 2 shows the performance of the models to replicate tour flows and the bottom their ability to replicate OD flows. For the S-EM, the TD-EM, the S-FTS-3-A/B and the TD-FTS-3-A/B models tour flows are converted to OD flows using Eq. (17).

The first salient result from the analysis is the low performance of the GM, for which the MAPE reaches $112.6 \%$. In the case of the S-EM, the MAPE displays a value of $52.5 \%$ for the tour flows and $44.3 \%$ for the OD flows, revealing a fair performance. For S-FTS-3-A the error metrics are slightly better than the ones displayed by the S-EM. For S-FTS-3-B, the results are significantly better, which reflect the importance given by DM B to the traffic counts.

When the metrics are computed by time interval, the error is higher for all the models. Once again, the lowest errors are found for the TD-FTS-3-B, where the MAPE ranges between $1 \%$ and $4.8 \%$ for the tour flows. When a single metric is computed 
Table 2

Performance of models considered.

\begin{tabular}{|c|c|c|c|c|c|c|c|c|c|}
\hline \multirow[t]{2}{*}{ Modeling approach } & \multirow{2}{*}{$\begin{array}{l}\text { MAPE for daily } \\
\text { flows estimates (\%) }\end{array}$} & \multirow{2}{*}{$\begin{array}{l}\text { Modeling } \\
\text { approach }\end{array}$} & \multicolumn{7}{|c|}{ MAPE for time intervals flows estimates } \\
\hline & & & AM $1(\%)$ & AM $2(\%)$ & AM $3(\%)$ & PM $1(\%)$ & PM $2(\%)$ & PM $3(\%)$ & Overall $^{*}(\%)$ \\
\hline \multicolumn{10}{|l|}{ Tour flows } \\
\hline S-EM & 52.5 & TD-EM & 44.1 & 49.2 & 55.3 & 54.7 & 47.8 & 57.0 & 51.2 \\
\hline S-FTS-3-A & 51.3 & TD-FTS-3-A & 36.3 & 28.1 & 27.0 & 25.7 & 24.5 & 23.7 & 29.1 \\
\hline S-FTS-3-B & 2.6 & TD-FTS-3-B & 2.9 & 4.8 & 1.0 & 2.2 & 4.2 & 1.0 & 2.5 \\
\hline \multicolumn{10}{|l|}{ OD Flows } \\
\hline DCGM & 112.6 & DCGM & 78.6 & 168.7 & 113.7 & 116.0 & 108.4 & 120.4 & 118.9 \\
\hline S-EM & 44.3 & TD-EM & 34.8 & 48.7 & 45.9 & 44.7 & 42.1 & 44.7 & 43.8 \\
\hline S-FTS-3-A & 43.0 & TD-FTS-3-A & 27.5 & 22.1 & 19.0 & 17.4 & 18.4 & 17.4 & 21.9 \\
\hline S-FTS-3-B & 1.0 & TD-FTS-3-B & 1.0 & 1.0 & 1.0 & 1.0 & 1.0 & 1.0 & 1.0 \\
\hline
\end{tabular}

Notes: (1) S-FTS-3 is applied to estimate daily flows. (2) S/TD-FTS-3-A uses the utility function of DM A; S/TD-FTS-3-B uses the utility function of DM B. (3) Overall $^{*}$ MAPE are computed for all the tour flows differentiated by departure time.

for all the time-dependent flows (last column on the right), the MAPE is $51.2 \%, 29.1 \%$, and 2.5\% for TD-EM, TD-FTS-3-A, and TD-FTS-3-B, respectively. In essence, TD-FTS-3-A and TD-FTS-3-B outperform S-EM and the GM in almost every case.

An important feature of aggregate methods is their ability to capture Trip Length Distribution (TLD). To gain some insight on the TLDs, the authors grouped trips frequency (i.e., commercial vehicles flow) into different travel costs bins. The results are shown Fig. 3.

As shown, the TLD can be reasonably approximated using a negative exponential model. The $R^{2}$ coefficient for the adjusted curve reveals a good fit (0.89). The average length of the tours is $138 \mathrm{~min}$, and the median $120 \mathrm{~min}$; around $12 \%$ of the tours take more than $4 \mathrm{~h}$, meaning that they start in one time interval and come back to the home base in a different interval.

To study the performance of the TD-FTS-3-A and TD-FTS-3-B in more detail, the resulting tour flows were plotted against the actual values, see Fig. 4. Each quadrant represents a time interval of departure time, and each dot represents the flow in one of the 4518 tour sequences. There is a good match between actual tour flows and estimated tour flows. As shown, tours departing during peak hours (AM 3, PM 1, and PM 2) tend to have the largest flows. In terms of the model performance, the results show that both TD-FTS-3 reproduce very closely the tour flows starting during peak hours, but tend to underestimate the flow for tour sequences starting during off hours. In general, the high values for $\mathrm{R}^{2}$ and the coefficients close to one for the trendline confirm the overall good performance of TD-FTS-3 models.

In terms of the flow distribution per industry sector, the TD-FTS-3-A result in an overall root mean square error (RMSE) of 0.76; and the shares of the different industry sector flows are reproduced closely. Fig. 5 shows the TD-FTS-3-A OD flow estimates for some industry sectors against the actual flows.

As shown, the TD-FTS-3-A model reproduces properly each industry flow. This is a key practical result because it allows the model to assess how changes in demand patterns of an industry sector affects the vehicles flow on the network. Another interesting result is that most of the simulated tour sequences were assigned a null (or close to zero) flow.

For forecasting purposes, the key outputs of the model are the Lagrange multipliers associated with each constraint. To obtain parameters with a unit (i.e., entropy) that does not depend on traffic counts, the TD-FTS-4 is used to recalibrate the parameters. It is important to note that the application of the TD-FTS- 4 resulted in flows that differ by less than $1 \%$ from the ones estimated by TD-FTS-3, thus all the results from TD-FTS-3 are also valid for TD-FTS-4. Table 3 shows the Lagrange multipliers associated with the cost constraint $(\beta)$ and the range of multipliers associated with the trip-ends constraints $(\lambda)$. As shown, $\beta$ is negative in all cases; this is conceptually valid as large costs for the tour or the OD trip should be associated with

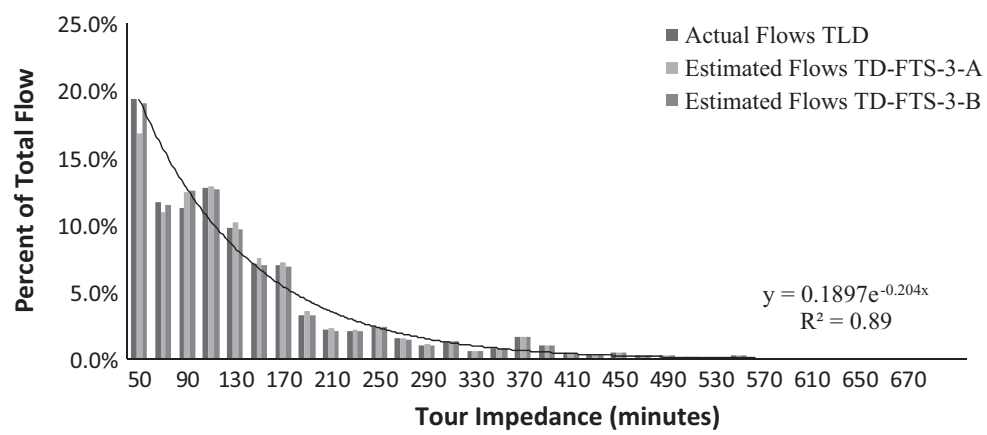

Fig. 3. Tour flow vs. tour impedance. Note: Correlation (actual flows, tours impedance) $=-0.80$. 

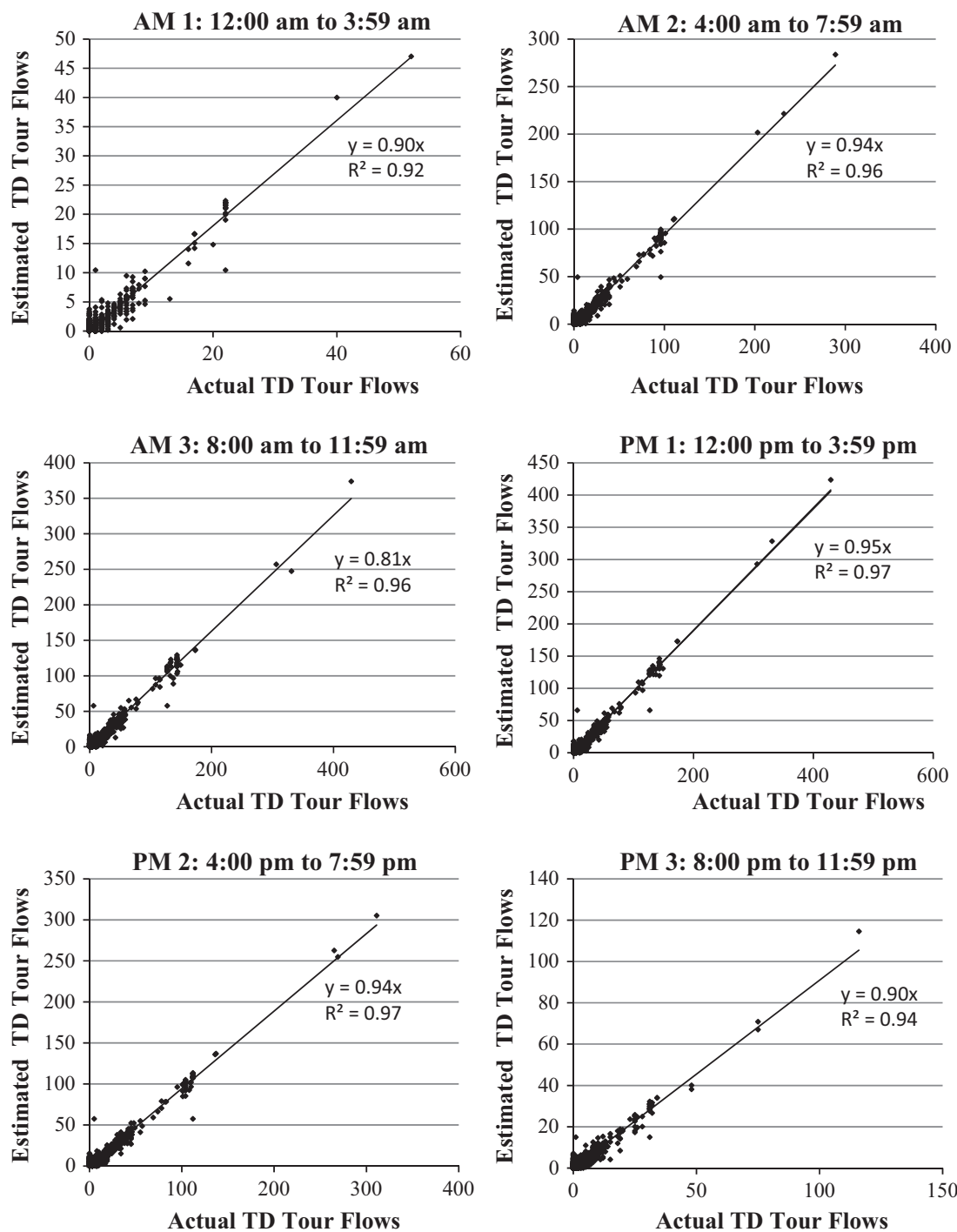

Fig. 4. Actual tour flows vs. TD-FTS-3-A estimated tour flows.

small flows. For the GM and S-EM the value of $\beta$ is -0.04233 and -0.00674 respectively. For the S-FTS-4-A and S-FTS-4-B, $\beta$ takes the value of -0.00697 and -0.04797 respectively, and for the TD-FTS-4-A and TD-FTS-4-B it takes the value of -0.00261 and -0.00829 respectively. The difference for the S-FTS- 4 and the TD-FTS- 4 cases implies that the cost of average distance is smaller when flows are differentiated by their departure time. In essence, the application of the TD-FTS adds a

Table 3

Lagrange multipliers for cost constraints.

\begin{tabular}{|c|c|c|c|c|c|}
\hline \multirow{2}{*}{$\begin{array}{l}\text { Modeling } \\
\text { approach }\end{array}$} & \multicolumn{2}{|l|}{ Daily flows estimates } & \multirow{2}{*}{$\begin{array}{l}\text { Modeling } \\
\text { approach }\end{array}$} & \multicolumn{2}{|l|}{ Time intervals } \\
\hline & $\begin{array}{l}\text { Lagrange multiplier } \\
\text { for cost }\end{array}$ & $\begin{array}{l}\text { Lagrange multiplier } \\
\text { for trip-ends }\end{array}$ & & $\begin{array}{l}\text { Lagrange multiplier } \\
\text { for cost }\end{array}$ & $\begin{array}{l}\text { Lagrange multiplier } \\
\text { for trip-ends }\end{array}$ \\
\hline \multicolumn{6}{|l|}{ Tour flows } \\
\hline S-EM & -0.00675 & From -13.9 to 19.2 & TD-EM & -0.00317 & From -15.8 to 20.8 \\
\hline S-FTS-4-A & -0.00697 & From -17.1 to 17.1 & TD-FTS-4-A & -0.00261 & From -16.6 to 16.6 \\
\hline S-FTS-4-B & -0.04797 & From -17.2 to 18.6 & TD-FTS-4-B & -0.00829 & From -14.5 to 17.7 \\
\hline \multicolumn{6}{|l|}{ OD flows } \\
\hline DCGM & -0.04233 & From 1.6 to 8.6 & n.a & n.a & n.a \\
\hline
\end{tabular}

Note: Penalty factor used for TD-FTS-4 is $p=100$. 

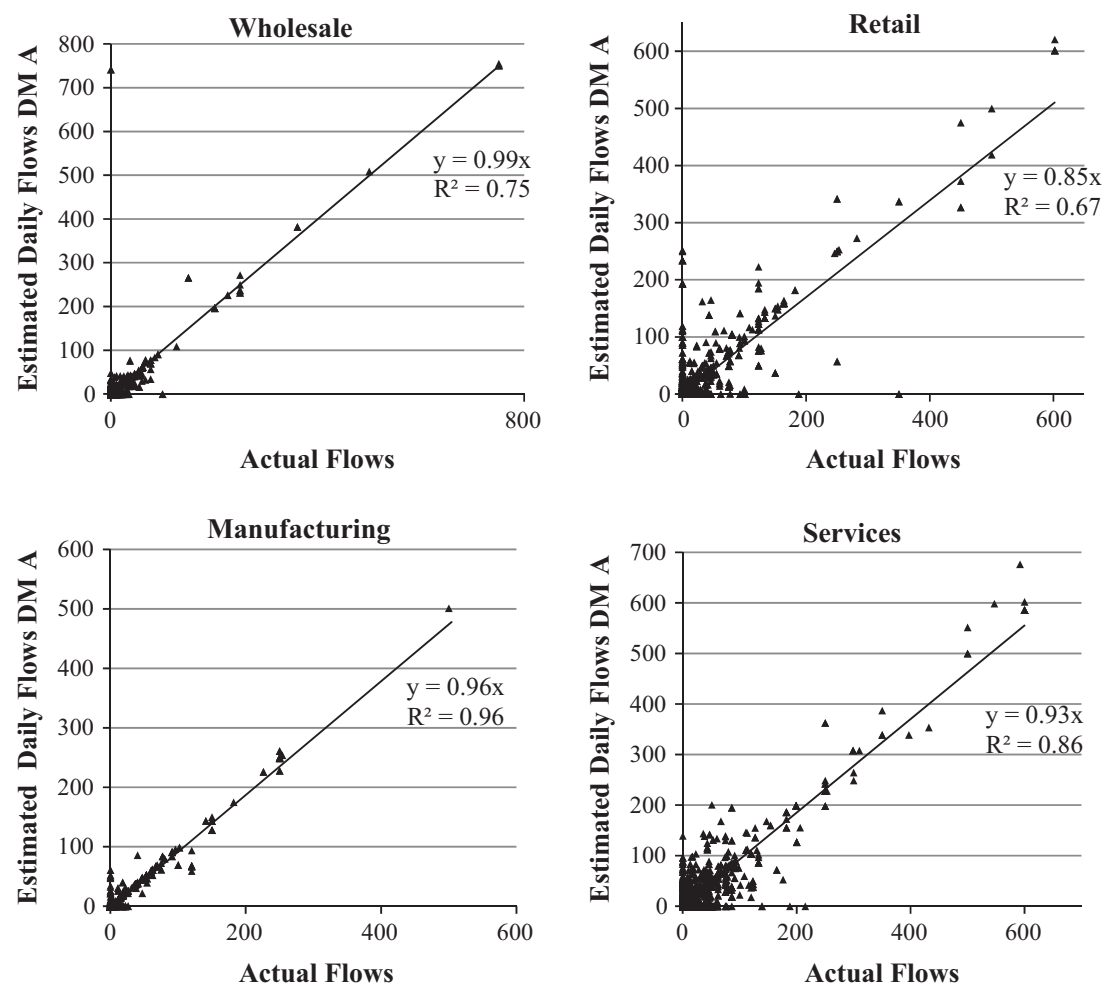

Fig. 5. Tour flows by industry sector for TD-FTS-3-A.

higher level of detail and incorporates the fact that travel cost is not only a matter of distance traveled (i.e., length of tour sequence) but also a consequence of departure time.

\subsection{Sensitivity analysis}

This section presents a sensitivity analysis for the different components affecting the performance of the TD-FTS-3. Part of the FTS models' performance can be attributed to the controlled setup and the way traffic counts were obtained. As traffic counts are affected by diverse factors (e.g., travel time variability, measurement errors), a sensitivity analysis is important to study how the variation of traffic counts affects the performance of TD-FTS-3-A and TD-FTS-3-B. To this effect, the variation of traffic counts was simulated for different scenarios using a lognormal distribution where the mean corresponds to a logarithmic transformation of the observed traffic, and the standard deviation is a function of the mean (e.g., 10\%, 30\%, 90\%). The box-and-whisker plot in Fig. 6 summarizes the results.

As shown in Fig. 6, although the median of the MAPE increases along with the error on the traffic counts, the median MAPE is lower than the one produced by the TD-EM (43.8\%) for every case. While for TD-FTS-3-A the median MAPE ranges between $22 \%$ and $38 \%$ for the different scenarios, for TD-FTS-3-B the median MAPE ranges between $8 \%$ and $39 \%$. These results show the model's success in incorporating the DM's preference structure to the utility as a function of entropy and traffic replication: DM A has a utility function that gives slightly more importance to traffic replication and therefore ends up with higher but steady MAPEs; while DM B has a nonlinear utility function that significantly favors traffic replication for low errors in traffic replication, but favors entropy for large errors. As a consequence, DM B ends up with very low MAPE for low variation in traffic counts, and higher MAPE for large variation but performing better than the TD-EM even in those cases. As shown, both TD-FTS-3 models tend to a MAPE of 39\% for large errors on traffic counts.

These results show that the TD-FTS-3 model is robust, though this finding should be confirmed in the future by testing stochastic traffic assignments, and a complete network where only few links' traffic counts are used as input for the model. Moreover, this sensitivity analysis can be implemented as part of the model, so that TD-FTS-3 provides an expected value for the tour flows and an interval of confidence.

Another parameter that plays a major role in the model is the total cost; this parameter should be estimated by the analyst based on other sources of information. As this estimation is prone to errors, the authors performed a sensitivity analysis to study how uncertainty on this parameter would affect the performance of the TD-FTS-3 models. The results indicate that, if a normal distribution with a $4 \%$ coefficient of variation is used to simulate uncertainty, the model produces in average 44.2\% MAPE with a standard deviation of $4.7 \%$ for TD-FTS-3-A, and a $33.1 \%$ MAPE with a standard deviation of $13.7 \%$ for 


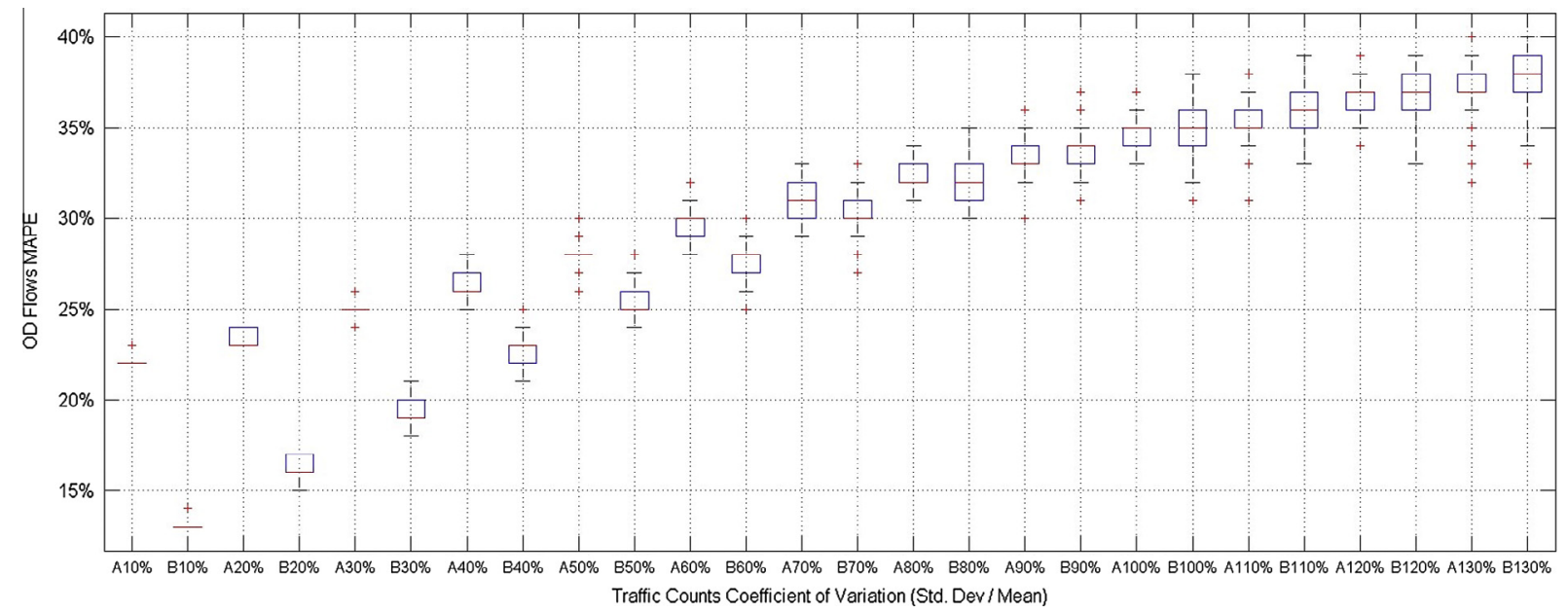

Fig. 6. Sensitivity Analysis for Variation of Traffic Counts. Note: The $x$-axis represents the traffic counts coefficient of variation, the letter before (i.e., A or B) represents the decision maker. The MAPEs are the result from applying 200 times the TD-FTS-3.

TD-FTS-3-B. These results show that TD-FTS-3 models are sensitive to the total cost; therefore, the analyst should give special attention to the estimation of total cost in the network. In essence, the time spent in these estimations is likely to be compensated with the model's reliability.

In the previous analysis, the TD-FTS-3 considered the traffic counts on the top 50\% super-links ranked by volume. As this coverage might not be always available, the authors assessed how different coverage scenarios would affect the performance of the model. The results showed that when the percentiles 50 and 75 of the super-links with higher volumes are covered, the results are similar to the full coverage scenario; but the best MAPE is reached only by the full coverage scenario. When only the percentile 90 and 95 are covered, the models have a lower performance and, naturally, tend to the value of the TD-EM. These results suggest that further research on the optimal coverage and location of traffic counts can enhance the effectiveness in the collection of secondary data.

\section{Conclusion}

The paper contributes to the research literature through the development of a tour-based time-dependent model for urban freight transport that is based on entropy maximization. This Time-Dependent Freight Tours Synthesis (TD-FTS) model can estimate the most likely set of tour flows, given a set of trip generation estimates, a set of time-dependent traffic counts and the total cost in the network. The resulting model TD-FTS- 2 is a bi-objective (i.e., the entropy function and the least squares function for traffic replication) convex program. One important contribution of this paper is to propose a methodology to incorporate the preference structure of the decision makers (DMs) using a multi-attribute utility function (MAV) to fuse the two objective functions. The model that incorporates the MAV is referred to as the TD-FTS- 3 and it is calibrated through an interview with the DM. The authors show the benefits of this approach compared to other techniques, such as the convex combination of objectives, the normalization, and the Pareto frontier. As the TD-FTS-3 depends on the traffic counts, the authors developed a recalibration process, referred to as TD-FTS-4. This model is used to calibrate a set of parameters that do not depend on traffic counts and that can be used for forecasting purposes. The authors also propose the TD-FTS-5 which is a model able to forecast time-dependent freight tour flows based on new freight trip generation estimates, on overall cost estimates for the network, and on the impedance parameter obtained from TD-FTS-4.

The data for the Denver Region were used as case study to assess the ability of the TD-FTS to replicate actual commercial vehicles flows, and to compare its performance with the Static Entropy Maximization model (S-EM) and the Gravity Model (GM). To apply the TD-FTS-2 model to the case study, the use of a Pareto frontier is a powerful pre-screening tool to analyze the tradeoffs between the two objectives of the model. However, it is necessary to find metrics that facilitate the interpretation of the objectives for the DM. The authors found that for this case study, the entropy and the traffic replication hold a strong relationship with the Mean Average Percentage Error (MAPE) and its standard deviation. The relationship can be exploited to interpret the DM preferences in terms of entropy and traffic replication. Using this information, a questionnaire was prepared to elicit DMs' preference structures and apply the MAV theory to derive utility function that represents the preference structure of the DM. The results from this process resulted into a linear function for a first DM (DM A) and a nonlinear function for a second DM (DM B). The functional form of the utility function and the magnitude of the coefficients had important implications on the TD-FTS performance.

The application of the models to the case study showed that, in general, the TD-FTS-3 models do a very good job in replicating the actual tour flows and the OD matrix. The TD-FTS-3 models outperform the GM and the S-EM in all metrics. The performance analysis revealed the TD-FTS-3 models' ability to reproduce the underlying flow patterns quite well; but also the potential 
errors of using a naïve approach to distribute S-EM or GM flows between time intervals. The results also showed the ability of the model to reproduce industry-based tour flows with an good performance, which opens a broad range of applications for city logistics. The recalibration process shows that the flows obtained from TD-FTS- 4 are very close to the ones from TD-FTS-3, indicating that the good performance for TD-FTS-3 also holds for TD-FTS-4. The results showed that the impedance parameter obtained from the TD-FTS-4 reproduces better the case study tour flows than the GM and the S-EM.

In terms of robustness, the model sensitivity to the quality of traffic counts depends directly on the utility function derived for each DM. The sensitivity analyses showed that the model would have an acceptable performance even when the estimated total cost varies, though if this variation is large feasibility issues will arise. Although a relaxation of the constraint imposed by the cost can solve the feasibility issue, the quality of the results may decrease significantly.

\subsection{Limitations}

As for every synthesis model, one of the main challenges is the lack of complete information to test the model. To overcome this problem, the authors used data from multiple sources (e.g., GPS, travel inventories, traffic studies) to construct the case study and complemented the missing information based on a number of assumptions, as described in Section 6 . Along with the advantages of this model, there are a number of limitations and opportunities for further research.

Although using tours as the modeling unit is a major contribution to the modeling literature, it also entails a number of challenges. The main one is to consider the dynamic nature of the tour formation process in the model. As tours are an outcome of operational decisions, the transportation analysis zones (TAZ) visited and the order on the tour sequence can change due to new conditions in the transportation system, as a result of market conditions, a different perception of travel times, time-windows imposed by customers, or due to many other reasons. This fluctuation cannot be entirely integrated into the model. To account for this variability, the model complements observed tour sequences with a wide range of simulated feasible tour sequences. The resulting set of tour sequences is used as input and the entropy maximization objective assigns flow to the most likely tour sequences. However, the tour sequences used in the input cannot cover the whole spectrum of possible tours (which is a combinatorial function of the number of TAZs).

Modeling urban freight using vehicle tours as unit has a number of advantages, but also limits the ability of the model to account for commodity flows, which are an important aspect in freight transportation. Further developments of the model should incorporate commodity flows to connect vehicle movements to the production-consumption links at the root of freight transportation. However, this also requires more research on urban commodity flows, which is still lacking.

In terms of using traffic counts for calibration, although their use overcomes the need for expensive data collection they also introduce an additional source of uncertainty to the model. Traffic conditions are a consequence of user choices and depend on a number of factors that include congestion, traffic disruptions, perception, and habit, among others. Although in the FTS model the importance of traffic counts for flow distribution depends on the preference structure derived from to the DM, using more sophisticated traffic assignment algorithms can improve the robustness of the model.

Further research will focus on the enhancement of the TD-FTS formulations to overcome these limitations. However, the findings presented in this paper imply that the TD-FTS can be used as a cost-effective method to reproduce accurately freight vehicles flows. In essence, implementing the TD-FTS for urban planning is bound not only to reduce freight demand studies cost but also to improve their performance.

\section{Statement of contribution}

Freight Origin-Destination Synthesis models are likely to play an important role in the development of urban freight demand models in the near future, as they have a great potential to model urban freight flows with minimum data needs. However, the literature review identified only a handful of papers published on this topic, in which the two main gaps identified are the lack of models considering (1) time-dependency and (2) tour-based behavior. To address these methodological gaps, this paper introduces a time-dependent model of urban freight demand, referred as Time-Dependent Freight Tour Synthesis (TD-FTS), which incorporates the time-dependent tour-based behavior of freight vehicles. The TD-FTS model seeks to obtain the most likely tour flows, given the tour sequences, the trip-ends, the link traffic volumes and cost constraints. The model is based on Entropy Maximization Theory and proposes a multi-attribute value function to assess the tradeoffs among the two competing objectives in the bi-objective program (i.e., entropy maximization, and minimization of error in traffic estimation). The numerical experiments, using the Denver Region as a case study, confirm the great potential of the TD-FTS model to reproduce tour flows, reproduce temporal effects and incorporate the decision maker's (DM) preference structure. The TD-FTS model outperforms other traditional models, such as the gravity model (GM), in all metrics.

\section{Acknowledgements}

The authors would like to acknowledge the support from the Volvo Research and Educational Foundations (VREF) that funded the Urban Freight Platform (UFP) and the Center of Excellence for Sustainable Urban Freight Systems (CoE-SUFS) 
through its Future Urban Transport research program. The authors would like to also thank the DRCOG staff for graciously sharing their unique data. Their contributions to this research are both acknowledged and appreciated.

\section{Appendix A}

\section{A.1. First-order conditions}

The Lagrangean function of the formulation is:

$$
L\left(x_{m, y}^{d}, \lambda_{i y}, \beta\right)=-\alpha_{1} v_{1}\left(z_{1}(x)\right)-\alpha_{2} v_{2}\left(z_{2}(x)\right)+\sum_{i=1}^{N} \lambda_{i y}\left(D_{i y}-\sum_{d=1}^{K} \sum_{m=1}^{M} \gamma_{i m}^{k} x_{m, y}^{d}\right)+\beta\left(C-\sum_{d=1}^{K} \sum_{m=1}^{M} c_{m}^{d} x_{m}^{d}\right)
$$

The first order conditions, also named Karush-Kuhn-Tucker (KKT) conditions can be obtained by deriving the Lagrangean function with respect to the flow on tours, as follows:

$$
\frac{\partial L\left(x_{m, y}^{d}, \lambda_{i y}, \beta\right)}{\partial x_{m^{\prime} y^{\prime}}^{d^{\prime}}}=-\alpha_{1} \dot{v}_{1}\left(z_{1}(\mathbf{x})\right) \frac{\partial z_{1}(\mathbf{x})}{\partial x_{m^{\prime} y^{\prime}}^{d^{\prime}}}-\alpha_{2} \dot{v}_{2}\left(z_{2}(\mathbf{x})\right) \frac{\partial z_{2}(\mathbf{x})}{\partial x_{m^{\prime} y^{\prime}}^{d^{\prime}}}-\sum_{i=1}^{N} \lambda_{i} \gamma_{i m}-\beta c_{m}^{d} \quad \forall m^{\prime} \in M, d^{\prime} \in K, y^{\prime} \in Y
$$

The derivative of the traffic replication and the entropy function can be expressed as:

$$
\begin{aligned}
& \frac{\partial z_{1}(\mathbf{x})}{\partial x_{m^{\prime} y^{\prime}}^{d^{\prime}}}=2 \sum_{k=1}^{K} \sum_{a=1}^{A}\left(\left(p_{m^{\prime}}^{a} \delta_{a m^{\prime}}^{k d^{\prime}}\right)\left(v_{a}^{k}-\sum_{d=1}^{K} \sum_{m=1}^{M} \sum_{y=1}^{Y} p_{m}^{a} \delta_{a m^{k}}^{k d} x_{m^{\prime} y}^{d}\right)\right) \quad \forall m^{\prime} \in M, d^{\prime} \in K, y^{\prime} \in Y \\
& \frac{\partial z_{2}(\mathbf{x})}{\partial x_{m^{\prime} y^{\prime}}^{d^{\prime}}}=\ln \left(x_{m^{\prime}, y^{\prime}}^{d^{\prime}}+\frac{1}{2}\right)-1 \quad \forall m^{\prime} \in M, d^{\prime} \in K, y^{\prime} \in Y
\end{aligned}
$$

The KKT conditions to ensure optimality are as follows:

$$
\begin{aligned}
& x_{m^{\prime}, y^{\prime}}^{d^{\prime}} \frac{\partial L\left(x_{m, y}^{d}, \lambda_{i y}, \beta\right)}{\partial x_{m^{\prime} y^{\prime}}^{d^{\prime}}}=0 \quad \forall m^{\prime} \in M, d^{\prime} \in K, y^{\prime} \in Y \\
& \frac{\partial L\left(x_{m, y}^{d}, \lambda_{i y}, \beta\right)}{\partial x_{m^{\prime}, y^{\prime}}^{d^{\prime}}} \geqslant 0 \quad \forall m^{\prime} \in M, d^{\prime} \in K, y^{\prime} \in Y \\
& \frac{\partial L\left(x_{m, y}^{d}, \lambda_{i y}, \beta\right)}{\partial \lambda_{i^{\prime}, y^{\prime}}}=0 \quad \forall i^{\prime} \in N, y^{\prime} \in Y \\
& \frac{\partial L\left(x_{m, y}^{d}, \lambda_{i y}, \beta\right)}{\partial \beta}=0 \\
& x_{m^{\prime} y^{\prime}}^{d^{\prime}} \geqslant 0 \quad \forall m^{\prime} \in M, k^{\prime} \in K, y^{\prime} \in Y
\end{aligned}
$$

Eqs. (A.7), (A.8) and (A.9) can be replaced by the constraints of the original problem stated in Eqs. (13)-(15), and Eqs. (A.2), (A.3) and (A.4) can be replaced on Eqs. (A.5) and (A.6), to obtain the KKT conditions, which depend on the specification of the value functions $v_{1}$ and $v_{2}$. The KKT first-order conditions are as follows:

$$
\begin{aligned}
x_{m^{\prime}, y^{\prime}}^{d^{\prime}} \frac{\partial L\left(x_{m, y}^{d}, \lambda_{i y}, \beta\right)}{\partial x_{m^{\prime}, y^{\prime}}^{d^{\prime}}}= & x_{m^{\prime} y}^{d}\left(-\alpha_{1} \dot{v}_{1}\left(z_{1}(\mathbf{x})\right)\left[2 \sum_{k=1}^{K} \sum_{a=1}^{A}\left(\left(p_{m^{\prime}}^{a} \delta_{a m^{\prime}}^{k d^{\prime}}\right)\left(v_{a}^{k}-\sum_{d=1}^{K} \sum_{m=1}^{M} \sum_{y=1}^{Y} p_{m}^{a} \delta_{a m}^{k d} x_{m^{\prime} y}^{d}\right)\right)\right]\right. \\
& \left.-\alpha_{2} \dot{v}_{2}\left(z_{2}(\mathbf{x})\right)\left(\ln \left(x_{m^{\prime}, y^{\prime}}^{d^{\prime}}+\frac{1}{2}\right)-1\right)-\sum_{i=1}^{N} \lambda_{i} \gamma_{i m}-\beta c_{m}^{d}\right)=0 \quad \forall m^{\prime} \in M, d^{\prime} \in K, y^{\prime} \in Y \\
\frac{\partial L\left(x_{m, y}^{d}, \lambda_{i, y}, \beta\right)}{\partial x_{m^{\prime}, y^{\prime}}^{d^{\prime}}}= & -\alpha_{1} \dot{v}_{1}\left(z_{1}(\mathbf{x})\right)\left[2 \sum_{k=1}^{K} \sum_{a=1}^{A}\left(\left(p_{m^{\prime}}^{a} \delta_{a m^{\prime}}^{k d^{\prime}}\right)\left(v_{a}^{k}-\sum_{d=1}^{K} \sum_{m=1}^{M} \sum_{y=1}^{Y} p_{m}^{a} \delta_{a m}^{k d} x_{m^{\prime} y}^{d}\right)\right)\right]-\alpha_{2} \dot{v}_{2}\left(z_{2}(\mathbf{x})\right)\left(\ln \left(x_{m^{\prime}, y^{\prime}}^{d^{\prime}}+\frac{1}{2}\right)-1\right) \\
& -\sum_{i=1}^{N} \lambda_{i} \gamma_{i m}-\beta c_{m}^{d} \geqslant 0 \quad \forall m^{\prime} \in M, d^{\prime} \in K, y^{\prime} \in Y
\end{aligned}
$$

$$
\sum_{d=1}^{K} \sum_{m=1}^{M} \gamma_{j m} x_{m, y}^{d}=D_{j}^{y} \quad \forall j \in N, y \in Y
$$




$$
\begin{aligned}
& \sum_{d=1}^{K} \sum_{m=1}^{M} \sum_{y=1}^{Y} c_{m}^{d} x_{m, y}^{d}=C \\
& x_{m^{\prime}, y^{\prime}}^{d^{\prime}} \geqslant 0 \quad \forall m^{\prime} \in M, k^{\prime} \in K, y^{\prime} \in Y
\end{aligned}
$$

\section{A.2. Second-order conditions}

The second derivative of the Lagrangean function is derived as follows:

$$
\begin{aligned}
\frac{\partial^{2} L\left(x_{m, y}^{d}, \lambda_{i, y}, \beta\right)}{\partial^{2} x_{m^{\prime}, y^{\prime}}^{d^{\prime}}}=- & \alpha_{1}\left(\ddot{v}_{1}\left(z_{1}(\mathbf{x})\right)\left[\frac{\partial z_{1}(\mathbf{x})}{\partial x_{m^{\prime} y^{\prime}}^{d^{\prime}}}\right]^{2}+\dot{v}_{1}\left(z_{1}(\mathbf{x})\right) \frac{\partial^{2} z_{1}(\mathbf{x})}{\partial^{2} x_{m^{\prime} y^{\prime}}^{d^{\prime}}}\right)-\alpha_{2}\left(\ddot{v}_{2}\left(z_{2}(\mathbf{x})\right)\left[\frac{\partial z_{2}(\mathbf{x})}{\partial z_{m^{\prime} y^{\prime}}^{d^{\prime}}}\right]^{2}+\dot{v}_{2}\left(z_{2}(\mathbf{x})\right) \frac{\partial^{2} z_{2}(\mathbf{x})}{\partial^{2} x_{m^{\prime} y^{\prime}}^{d^{\prime}}}\right) \\
& \forall m^{\prime} \in M, d^{\prime} \in K, y^{\prime} \in Y
\end{aligned}
$$

where the Jacobian of the traffic replication and the entropy functions is defined in Eqs. (A.3) and (A.4), and their Hessian are:

$$
\begin{aligned}
& \frac{\partial z_{1}^{2}(\mathbf{x})}{\partial x_{m 1, y 1}^{d 1} \partial x_{m 2, y 2}^{d 2}}=\left\{\begin{array}{cc}
2 \sum_{k=1}^{K} \sum_{a=1}^{A}\left(p_{m^{\prime}}^{a} \delta_{a m}^{k d^{\prime}}\right)^{2} & \text { for } m_{1}=m_{2}, d_{1}=d_{2}, y_{1}=y_{2} \\
2 \sum_{k=1}^{K} \sum_{a=1}^{A}\left(p_{m 1}^{a} \delta_{a m 1}^{k d 1}\right)\left(p_{m 2}^{a} \delta_{a m 2}^{k d 2}\right) & \text { otherwise }
\end{array} \quad \forall m \in M, d \in K, y \in Y\right. \\
& \frac{\partial z_{2}^{2}(\mathbf{x})}{\partial x_{m 1, y 1}^{d 1} \partial x_{m 2, y 2}^{d 2}}=\left\{\begin{array}{cc}
\frac{1}{x_{m^{\prime} y}+\frac{1}{2}} & \text { for } m_{1}=m_{2}, d_{1}=d_{2}, y_{1}=y_{2} \\
0 & \text { otherwise }
\end{array} \quad \forall m \in M, d \in K, y \in Y\right.
\end{aligned}
$$

As shown in these equations, the symmetric structure of the matrix and its nonnegative components reveal that the Hessian of the entropy function is positive semi-definite. Therefore, this function is strictly convex. In terms of the Jacobian of the traffic replication function, it can be written in matrix form as:

$$
\nabla^{2} e(\mathbf{x})=2 \mathbf{P}^{T} \mathbf{P}
$$

where each component of $\mathbf{P}$ is defined by $p_{m^{\prime}}^{a} \delta_{a m}^{k d}$.

$\mathbf{P}^{T} \mathbf{P}$ is positive definite because $\mathbf{x}^{T} \mathbf{P P}^{T} \mathbf{x}=\left(\mathbf{P}^{T} \mathbf{x}\right)^{T}\left(\mathbf{P}^{T} \mathbf{x}\right)>0$.

Moreover, Eq. (A.15) shows that the second derivative of the Lagrangean depends on: the value functions, the traffic repli-

\begin{tabular}{|c|c|c|c|c|c|c|c|c|c|}
\hline Zone & Lagrange multiplier & Zone & Lagrange multiplier & Zone & Lagrange multiplier & Zone & Lagrange multiplier & Zone & Lagrange multiplier \\
\hline $\mathrm{Z1}$ & 3.05 & $\mathrm{Z} 51$ & -1.44 & Z102 & 4.87 & Z152 & 0.23 & Z202 & 4.94 \\
\hline $\mathrm{Z} 2$ & -0.71 & $\mathrm{Z} 52$ & 1.10 & Z103 & 5.23 & Z153 & 4.47 & Z203 & -1.60 \\
\hline $\mathrm{Z3}$ & 2.77 & $\mathrm{Z} 53$ & 26.34 & Z104 & 0.82 & Z154 & 3.64 & Z204 & 8.11 \\
\hline Z4 & 12.59 & $\mathrm{Z} 54$ & 0.29 & Z105 & 5.55 & Z155 & 3.63 & Z205 & 3.79 \\
\hline $\mathrm{Z} 5$ & 0.59 & $\mathrm{Z} 55$ & 0.22 & Z106 & -1.94 & Z156 & 3.47 & Z206 & -6.19 \\
\hline Z6 & 10.31 & Z56 & -1.73 & Z107 & 7.25 & Z157 & 1.23 & Z207 & 1.71 \\
\hline $\mathrm{Z7}$ & -0.31 & $\mathrm{Z} 57$ & 3.23 & Z108 & 3.64 & Z158 & -4.47 & Z208 & 8.33 \\
\hline Z8 & -1.91 & Z58 & 5.63 & Z109 & 0.85 & Z159 & 3.57 & Z209 & -2.85 \\
\hline Z9 & -8.04 & Z59 & -18.79 & Z110 & 4.44 & $\mathrm{Z} 160$ & -12.82 & $Z 210$ & 3.68 \\
\hline Z10 & 3.17 & Z60 & -18.74 & Z111 & -6.68 & Z161 & -11.81 & Z211 & 0.19 \\
\hline Z11 & -11.39 & Z61 & -7.22 & Z112 & 5.11 & Z162 & -23.11 & $\mathrm{Z} 212$ & -0.70 \\
\hline $\mathrm{Z} 12$ & -2.64 & Z62 & -3.12 & Z113 & 1.04 & Z163 & 0.00 & Z213 & 5.88 \\
\hline Z13 & 4.78 & Z63 & 6.12 & Z114 & 0.76 & Z164 & 1.84 & Z214 & -18.93 \\
\hline $\mathrm{Z14}$ & 2.90 & Z64 & 31.24 & Z115 & -3.41 & Z165 & 2.50 & Z215 & 2.43 \\
\hline Z15 & 4.05 & Z65 & 7.06 & Z116 & 2.39 & Z166 & -8.21 & Z216 & 16.48 \\
\hline
\end{tabular}
cation function, the entropy function, the Jacobian and the Hessian of these functions, and on the scale factors, so the final second-order conditions will depend on the MAV derived from the decision makers' preferences.

\section{Appendix B}

See Table 4.

Table 4

Example - Lagrange multipliers for each TAZ in S-EM. 
Table 4 (continued)

\begin{tabular}{|c|c|c|c|c|c|c|c|c|c|}
\hline Zone & Lagrange multiplier & Zone & Lagrange multiplier & Zone & Lagrange multiplier & Zone & Lagrange multiplier & Zone & Lagrange multiplier \\
\hline Z16 & 1.18 & Z66 & 5.17 & Z117 & 1.85 & Z167 & 2.20 & $\mathrm{Z} 217$ & 5.06 \\
\hline $\mathrm{Z} 17$ & 0.75 & Z67 & -13.34 & Z118 & 8.42 & Z168 & 3.06 & Z218 & -0.37 \\
\hline Z18 & 5.55 & Z68 & 8.74 & Z119 & 4.71 & Z169 & 0.63 & Z219 & 1.04 \\
\hline Z19 & 0.68 & Z69 & 20.79 & Z120 & 6.04 & $\mathrm{Z} 170$ & 3.87 & $\mathrm{Z} 220$ & -2.25 \\
\hline $\mathrm{Z} 20$ & 1.78 & $\mathrm{Z70}$ & 14.61 & Z121 & 2.43 & $\mathrm{Z} 171$ & 6.31 & $\mathrm{Z} 221$ & 0.58 \\
\hline $\mathrm{Z} 21$ & 11.78 & Z71 & -3.47 & Z122 & 3.48 & $\mathrm{Z} 172$ & -5.36 & Z222 & 0.05 \\
\hline $\mathrm{Z} 22$ & 5.23 & $\mathrm{Z72}$ & -9.23 & Z123 & 6.62 & Z173 & -6.86 & Z223 & 1.60 \\
\hline Z23 & 4.96 & Z73 & 7.65 & Z124 & 2.58 & Z174 & 0.17 & Z224 & 0.81 \\
\hline $\mathrm{Z} 24$ & 5.25 & $\mathrm{Z74}$ & 1.35 & Z125 & -1.81 & Z175 & 8.83 & Z225 & 2.36 \\
\hline $\mathrm{Z} 25$ & 0.01 & $\mathrm{Z75}$ & -5.29 & Z126 & -2.40 & Z176 & 9.51 & Z226 & 0.04 \\
\hline Z26 & -15.55 & Z76 & 3.81 & Z127 & 2.97 & Z177 & 13.73 & $\mathrm{Z} 227$ & 1.73 \\
\hline $\mathrm{Z} 27$ & 0.00 & $\mathrm{Z77}$ & -1.29 & Z128 & 5.21 & Z178 & 0.00 & Z228 & -0.24 \\
\hline $\mathrm{Z} 28$ & 1.56 & Z78 & 27.75 & Z129 & 1.49 & Z179 & -5.78 & Z229 & -13.60 \\
\hline Z29 & 3.83 & Z79 & -3.05 & Z130 & 1.85 & $\mathrm{Z} 180$ & -4.12 & $\mathrm{Z} 230$ & 1.64 \\
\hline $\mathrm{Z30}$ & 1.75 & $\mathrm{Z} 80$ & 27.67 & Z131 & 3.02 & Z181 & 6.85 & Z231 & 2.54 \\
\hline Z31 & -4.47 & Z81 & 0.00 & Z132 & 1.49 & Z182 & 2.22 & Z232 & 2.84 \\
\hline Z32 & 2.70 & Z82 & -26.05 & Z133 & 2.34 & Z183 & 10.05 & Z233 & -1.34 \\
\hline Z33 & 7.11 & Z83 & 3.63 & Z134 & 4.07 & Z184 & -21.36 & Z234 & 0.10 \\
\hline Z34 & 0.00 & Z84 & 1.39 & Z135 & 0.00 & Z185 & 9.61 & Z235 & -5.50 \\
\hline Z35 & -0.55 & Z85 & -17.88 & Z136 & 15.74 & Z186 & 5.32 & Z236 & 1.58 \\
\hline Z36 & -0.08 & Z86 & 2.09 & Z137 & 0.67 & Z187 & -2.50 & Z237 & 2.41 \\
\hline Z37 & 7.00 & Z87 & 3.96 & Z138 & 0.34 & Z188 & 0.00 & Z238 & -0.88 \\
\hline Z38 & 2.55 & Z88 & 4.98 & Z139 & 5.40 & Z189 & 18.08 & Z239 & 0.00 \\
\hline Z39 & -5.94 & Z89 & 0.84 & $\mathrm{Z} 140$ & 0.69 & Z190 & -7.17 & $\mathrm{Z} 240$ & -8.83 \\
\hline $\mathrm{Z} 40$ & -0.29 & Z90 & 3.84 & Z141 & 8.53 & Z191 & 3.65 & Z241 & -1.81 \\
\hline Z41 & -4.52 & Z91 & 0.00 & Z142 & 0.74 & Z192 & 0.46 & Z242 & 4.08 \\
\hline Z42 & -17.54 & Z92 & 6.32 & Z143 & 0.79 & Z193 & -2.24 & Z243 & -10.71 \\
\hline Z43 & -3.89 & Z93 & 33.22 & Z144 & 0.67 & Z194 & -1.14 & $\mathrm{Z} 244$ & 2.32 \\
\hline Z44 & 21.20 & Z94 & 1.75 & Z145 & 1.05 & Z195 & 1.40 & Z245 & 1.24 \\
\hline $\mathrm{Z} 45$ & 3.38 & Z96 & 5.97 & Z146 & 8.36 & Z196 & -8.61 & Z246 & -0.24 \\
\hline Z46 & 2.16 & Z97 & 5.70 & Z147 & 0.21 & Z197 & 3.64 & $\mathrm{Z} 247$ & 0.74 \\
\hline $\mathrm{Z} 47$ & 8.63 & Z98 & 7.11 & Z148 & -3.53 & Z198 & -5.77 & Z248 & 1.93 \\
\hline Z48 & 16.24 & Z99 & -1.28 & Z149 & 0.00 & Z199 & -53.11 & Z249 & -2.51 \\
\hline Z49 & 2.73 & Z100 & 2.96 & Z150 & 0.30 & Z200 & -3.64 & $\mathrm{Z} 250$ & 3.64 \\
\hline $\mathrm{Z} 50$ & -10.11 & Z101 & 2.33 & Z151 & 6.40 & Z201 & 2.85 & Z251 & 2.74 \\
\hline Z252 & -1.56 & Z302 & 1.73 & Z352 & 3.88 & Z402 & 1.17 & Z452 & 2.97 \\
\hline Z253 & 2.64 & Z303 & -7.22 & Z353 & -1.08 & Z403 & -0.97 & Z453 & 0.00 \\
\hline Z254 & 4.78 & Z304 & 7.86 & Z354 & -2.70 & Z404 & -2.82 & Z454 & 0.00 \\
\hline Z255 & 18.71 & Z305 & -7.50 & Z355 & 0.00 & Z405 & 19.05 & Z455 & 14.03 \\
\hline Z256 & 6.27 & Z306 & 0.00 & Z356 & 3.72 & Z406 & 1.66 & Z456 & 0.38 \\
\hline Z257 & 2.68 & Z307 & 2.19 & Z357 & -2.64 & Z407 & 4.58 & Z457 & 2.49 \\
\hline Z258 & 3.59 & Z308 & 1.70 & Z358 & 2.03 & Z408 & -11.89 & Z458 & 0.00 \\
\hline Z259 & 4.16 & Z309 & 5.72 & Z359 & 12.94 & Z409 & 0.00 & Z459 & 6.16 \\
\hline $\mathrm{Z} 260$ & -1.32 & Z310 & 3.10 & $\mathrm{Z360}$ & -2.97 & Z410 & -16.40 & $\mathrm{Z} 460$ & -0.86 \\
\hline Z261 & 2.13 & Z311 & 1.99 & Z361 & 4.44 & Z411 & 7.52 & Z461 & -5.52 \\
\hline Z262 & 2.98 & Z312 & 5.85 & Z362 & 11.11 & Z412 & 0.00 & Z462 & 1.73 \\
\hline Z263 & 2.09 & Z313 & 4.65 & Z363 & 15.96 & Z413 & 1.28 & Z463 & -0.84 \\
\hline Z264 & 3.05 & Z314 & 7.58 & Z364 & 11.68 & Z414 & -1.00 & Z464 & 6.36 \\
\hline Z265 & 2.06 & Z315 & 6.97 & Z365 & 7.37 & Z415 & 3.07 & Z465 & 5.01 \\
\hline Z266 & 0.00 & Z316 & -2.88 & Z366 & 3.70 & Z416 & 4.13 & Z466 & 0.00 \\
\hline Z267 & 3.90 & Z317 & -1.48 & Z367 & -5.81 & Z417 & 8.42 & Z467 & 0.00 \\
\hline Z268 & -2.05 & Z318 & -18.03 & Z368 & -2.00 & Z418 & 12.44 & Z468 & -2.48 \\
\hline Z269 & 0.00 & Z319 & 2.65 & Z369 & 0.00 & Z419 & 3.28 & Z469 & 0.00 \\
\hline $\mathrm{Z} 270$ & -5.32 & Z320 & -17.11 & Z370 & 0.00 & Z420 & 2.12 & $\mathrm{Z} 470$ & 2.99 \\
\hline Z271 & 1.82 & Z321 & 0.00 & Z371 & -6.96 & Z421 & 8.73 & Z471 & 0.07 \\
\hline Z272 & 3.18 & Z322 & 4.08 & Z372 & 2.66 & Z422 & 0.00 & Z472 & -3.20 \\
\hline Z273 & 1.81 & Z323 & 5.31 & Z373 & -8.00 & Z423 & 5.04 & Z473 & 31.45 \\
\hline Z274 & 6.50 & Z324 & 3.78 & Z374 & -8.65 & Z424 & 3.72 & Z474 & 0.00 \\
\hline Z275 & 4.18 & Z325 & 6.27 & Z375 & -4.18 & Z425 & -3.58 & Z475 & 0.46 \\
\hline Z276 & 8.39 & Z326 & 5.95 & Z376 & 0.00 & Z426 & -1.60 & Z476 & 0.00 \\
\hline Z277 & 2.64 & Z327 & 3.11 & Z377 & -34.94 & Z427 & 7.95 & Z477 & -29.73 \\
\hline Z278 & 1.16 & Z328 & 4.78 & Z378 & 0.00 & Z428 & -0.69 & Z478 & 13.04 \\
\hline Z279 & -0.83 & Z329 & -23.39 & Z379 & 0.00 & Z429 & 0.62 & Z479 & 0.00 \\
\hline $\mathrm{Z} 280$ & 2.58 & Z330 & 33.84 & $\mathrm{Z380}$ & 0.00 & Z430 & 7.89 & $\mathrm{Z} 480$ & 3.02 \\
\hline Z281 & -1.98 & Z331 & 4.84 & Z381 & 0.00 & Z431 & -3.74 & Z481 & 0.00 \\
\hline Z282 & 0.00 & Z332 & -3.60 & Z382 & 0.00 & Z432 & 6.11 & Z482 & 0.00 \\
\hline Z283 & 3.12 & Z333 & 0.00 & Z383 & 1.88 & Z433 & 1.13 & Z483 & -0.10 \\
\hline Z284 & 1.18 & Z334 & 7.88 & Z384 & 1.24 & Z434 & 1.45 & & \\
\hline Z285 & 9.81 & Z335 & -4.36 & Z385 & 2.08 & Z435 & 7.58 & & \\
\hline
\end{tabular}


Table 4 (continued)

\begin{tabular}{|c|c|c|c|c|c|c|c|c|c|}
\hline Zone & Lagrange multiplier & Zone & Lagrange multiplier & Zone & Lagrange multiplier & Zone & Lagrange multiplier & Zone & Lagrange multiplier \\
\hline Z286 & 4.19 & Z336 & 10.20 & Z386 & 1.14 & Z436 & 1.94 & & \\
\hline Z287 & 5.15 & Z337 & -18.04 & Z387 & -6.12 & Z437 & 3.81 & & \\
\hline Z288 & 0.00 & Z338 & 3.56 & Z388 & 0.00 & Z438 & -0.65 & & \\
\hline Z289 & -19.97 & Z339 & 4.05 & Z389 & 1.44 & Z439 & 2.95 & & \\
\hline Z290 & 0.00 & Z340 & 0.76 & Z390 & -5.89 & $\mathrm{Z} 440$ & 0.21 & & \\
\hline Z291 & 0.51 & Z341 & -5.98 & Z391 & 1.59 & Z441 & 1.46 & & \\
\hline Z292 & 6.48 & Z342 & 0.73 & Z392 & 7.26 & Z442 & 0.00 & & \\
\hline Z293 & -3.65 & Z343 & 3.19 & Z393 & -0.62 & Z443 & 1.15 & & \\
\hline Z294 & 1.36 & Z344 & 2.03 & Z394 & 14.66 & Z444 & 3.68 & & \\
\hline Z295 & 5.43 & Z345 & 1.31 & Z395 & -3.89 & Z445 & 0.00 & & \\
\hline Z296 & 3.03 & Z346 & -2.97 & Z396 & -1.11 & Z446 & 4.33 & & \\
\hline Z297 & 2.00 & Z347 & 1.83 & Z397 & -0.39 & Z447 & -6.91 & & \\
\hline Z298 & 8.44 & Z348 & 0.88 & Z398 & 2.58 & Z448 & 0.00 & & \\
\hline Z299 & 1.21 & Z349 & -11.96 & Z399 & 4.92 & Z449 & 4.18 & & \\
\hline Z300 & 3.08 & Z350 & 1.24 & $\mathrm{Z} 400$ & 2.94 & $\mathrm{Z} 450$ & -1.62 & & \\
\hline Z301 & 3.65 & Z351 & 1.17 & Z401 & 7.01 & Z451 & 5.19 & & \\
\hline
\end{tabular}

\section{References}

Al-Battaineh, O., Kaysi, I., 2005. Commodity-based truck origin-destination matrix estimation using input-output data and genetic algorithms. Transportation Research Record: Journal of the Transportation Research Board 1923 (-1), 37-45.

Al-Battaineh, O., Kaysi, I.A., 2005. Commodity-based truck origin-destination matrix estimation using input-output data and genetic algorithms. Transportation Research Record: Journal of the Transportation Research Board 1923 (1), 37-45.

Bera, S., Rao, K.V.K., 2011. Estimation of origin-destination matrix from traffic counts: the state of the art. European Transport/Trasporti Europei 49, $2-23$. Boerkamps, J., Binsbergen, A.V., 1999. GoodTrip - A New Approach for Modelling and Evaluation of Urban Goods Distribution. City Logistics I, Cairns, Australia.

Brooke, A., Rosenthal, R.E., 2003. GAMS. GAMS Development Corporation, Washington, D.C..

Burnside, W., 1917. A rapidly convergent series for log N. Messenger Math 46, 157-159.

Catton, W.R., 1982. Overshoot: The Ecological Basis of Revolutionary Change. IL, University of Illinois Press, Champaign.

Chankong, V., Haimes, Y.Y., 1983. Multiobjective Decision Making: Theory and Methodology. Elsevier Science Publishing Co., Inc., New York, USA.

de Grange, L., González, F., 2012. Mejorando la aproximacion de Stirling en modelos de distribucion de viajes. XV Congreso Panamericano de Ingeniería de Transporte, Santiago, Chile.

Denver Regional Council of Governments and Parsons Transportation Group Inc., 2001. Commercial Vehicle Survey Report. Denver, Colorado.

Donnelly, R., 2007. A hybrid microsimulation model of freight flows. In: Taniguchi, E., Thompson, R.G. (Eds.), Proceedings of the 4th International Conference on City Logistics, July 11-13, 2007. Institute for City Logistics, Crete, Greece, pp. 235-246.

Franklin, G.F., Workman, M.L., Powell, D., 1997. Digital Control of Dynamic Systems. Addison-Wesley Longman Publishing Co., Inc., Boston, MA, USA.

Gedeon, C., Florian, M., Crainic, T., 1993. Determining origin-destination matrices and optimal multiproduct flows for freight transportation over multimodal networks. Transportation Research Part B 27B (5), 351-368.

Gédéon, C., Florian, M., Crainic, T.G., 1993. Determining origin-destination matrices and optimal multiproduct flows for freight transportation over multimodal networks. Transportation Research Part B 27 (5), 351-368.

Holguín-Veras, J., 2000. A framework for an integrative freight market simulation. In: IEEE 3rd Annual Intelligent Transportation Systems Conference ITSC2000, Dearborn, Michigan, IEEE.

Holguín-Veras, J., Aros-Vera, F., 2014. Self-supported freight demand management: pricing and incentives. EURO Journal on Transportation and Logistics 3 (1), 1-24.

Holguín-Veras, J., Jaller, M., 2014. Comprehensive freight demand data collection framework for large urban areas. In: González-Feliu, J., Semet, F., Routhier, J.L. (Eds.), Sustainable Irban Logistics: Concepts, Methods and Information Systems. Springer-Verlag, Berlin.

Holguín-Veras, J., Patil, G., 2005. Observed trip chain behavior of commercial vehicles. Transportation Research Record 1906, 74-80.

Holguín-Veras, J., Patil, G., 2007. Integrated origin-destination synthesis model for freight with commodity based and empty trip model. Transportation Research Record: Journal of the Transportation Research Board 2008, 60-66.

Holguín-Veras, J., Patil, G., 2008. A multicommodity integrated freight origin-destination synthesis model. Networks and Spatial Economics 8 (2), 309-326.

Holguín-Veras, J., Thorson, E., 2000. An investigation of the relationships between the trip length distributions in commodity-based and trip-based freight demand modeling. Transportation Research Record 1707, 37-48.

Holguín-Veras, J., Wang, Q., Xu, N., Ozbay, K., Cetin, M., Polimeni, J., 2006. Impacts of time of day pricing on the behavior of freight carriers in a congested urban area: implications to road pricing. Transportation Research Part A 40 (9), 744-766.

Holguín-Veras, J., Ban, J., Jaller, M., Destro, L., Marquis, R., 2010. Feasibility Study for Freight Data Collection, Final report, Rensselaer Polytechnic Institute, Albany, NY, pp. 1-231.

Holguín-Veras, J., Jaller, M., Sanchez-Díaz, I., Wojtowicz, J.M., Campbell, S., Levinson, H.S., Lawson, C.T., Powers, E., Tavasszy, L., 2012. NCHRP Report 739/ NCFRP Report 19: Freight Trip Generation and Land Use. National Cooperative Highway Research Program/National Cooperative Freight Research Program, , vol. 165, Transportation Research Board of the National Academies, Washington, DC.

Holguín-Veras, J., Thorson, E., Wang, Q., Xu, N., González-Calderón, C., Sánchez-Díaz, I., Mitchell, J., 2013. Urban freight tour models: state of the art and practice. In: Ben-Akiva, M., Meersman, H., Van de Voorde, E. (Eds.), Freight Transport Modelling. Emerald Group Publishing Limited, UK, pp. $335-352$.

Hunt, J.D., Stefan, K.J., 2007. Tour-based microsimulation of urban commercial movements. Transportation Research Part B 41 (9), $981-1013$.

Jones, C., Murray, D., Short, J., 2005. Methods of travel time measurement in freight-significant corridors. In: 84th Annual Meeting of Transportation Research Board.

Keeney, R.L., Raiffa, H., 1993. Tradeoffs Under Certainty. Decisions with Multiple Objectives: Preferences and Value Tradeoffs, vol. 569. Cambridge Univ Pr.

Lawson, C., Holguín-Veras, J., Sánchez-Díaz, I., Jaller, M., Campbell, S., Powers, E., 2012. Estimated generation of freight trips based on land use. Transportation Research Record: Journal of the Transportation Research Board 2269, 65-72.

Liedtke, G., 2006. An Actor-Based Approach to Commodity Transport Modelling. PhD, Karlsruhe Universität.

Liedtke, G., 2009. Principles of micro-behavior commodity transport modeling. Transportation Research Part E 45 (5), 795-809.

Liedtke, G., Schepperle, H., 2004. Segmentation of the transportation market with regard to activity-based freight transport modelling. International Journal of Logistics Research and Applications: A Leading Journal of Supply Chain Management 7 (3), 199-218. 
List, G.F., Turnquist, M.A., 1994a. Estimating truck travel patterns in urban areas. Transportation Research Record: Journal of the Transportation Research Board 1430, 1-9.

List, G.F., Turnquist, M.A., 1994b. Estimating Truck Travel Patterns in Urban Areas. ETATS-UNIS, National Research Council, Washington, DC.

Ma, Y., van Zuylen, H., Kuik, R., 2012. Freight origin-destination estimation using Bayesian networks based on multiple data sources. In: 4th World Conference P\&OM, Amsterdam, The Netherlands.

Noortman, H.J., van Es, J., 1978. Traffic model. Manuscript for the Dutch Freight Transport Model.

Nozick, L., Turnquist, M., List, G., 1996. Trade pattern estimation between the United States and Mexico. Transportation Research Circular: Transportation Research Board 459, 74-86.

Ortúzar, J.D., Willumsen, L.G., 2011. Modelling Transport. John Wiley and Sons, New York.

Pearce, E., 1957. History of the Standard Industrial Classification, Prepared for the Executive Office of the President. Bureau of the Budget.

Robillard, P., 1975. Estimating the O-D matrix from observed link volumes. Transportation Research 9 (2), $123-128$.

Rockafellar, R.T., Wets, R.J.B., 1998. Convexity. Variational Analysis 317, 38-76.

Routhier, J.L., Toilier, F., 2007. FRETURB V3, a policy oriented software tool for modelling urban goods movement. In: 11th World Conference on Transport Research, Berkeley, CA

Sanchez-Diaz, I., 2014. A Time-Dependent Freight Tour Synthesis Model - An Integrative Freight Demand Model, Rensselaer Polytechnic Institute.

Sanchez-Diaz, I., Holguin-Veras, J., Wang, X., 2014. An exploratory analysis of spatial effects on freight trip attraction. Transportation (in press). http:// dx.doi.org/10.1007/s11116-014-9570-1.

Silas, M.A., Holguín-Veras, J., 2009. Behavioral microsimulation formulation for analysis and design of off-hour delivery policies in urban areas. Transportation Research Record: Journal of the Transportation Research Board 2097, 43-50.

Stefan, K., McMillan, J., Hunt, J., 2005. Urban commercial vehicle movement model for Calgary, Alberta, Canada. Transportation Research Record: Journal of the Transportation Research Board 1921, 1-10.

Tamin, O.Z., Willumsen, L.G., 1988. Freight demand model estimation from traffic counts. In: PTRC Annual Meeting, University of Bath, England

Tamin, O.Z., Willumsen, L.G., 1992. Freight demand model estimation from traffic counts. In: PTRC Annual Meeting. England, University of Bath.

Tavasszy, L.A., Stada, J.E., Hamerslag, R., 1994. The impact of decreasing border barriers in Europe on freight transport flows by road. In: Proceedings of the 36th Annual Conference of the Transportation Research Forum, Florida, USA.

Thorson, E., 2005. The Integrative Freight Market Simulation: An Application of Experimental Economics and Algorithmic Solutions. Ph.D., Rensselaer Polytechnic Institute.

van Duin, J.H.R., Tavasszy, L.A., Taniguchi, E., 2007. Real time simulation of auctioning and re-scheduling processes in hybrid freight markets. Transportation Research Part B 41 (9), 1050-1066.

Van Zuylen, H.J., Willumsen, L.G., 1980. The most likely trip matrix estimated from traffic counts. Transportation Research Part B 14 (3), 281-293.

Vleugel, J.M., Janic, M., 2004. Route choice and the impact of 'logistics routes'. In: Taniguchi, E., Thompson, R., Logistics Systems for Sustainable Cities, Amsterdam, Elsevier, pp. 221-233.

Wang, Q., Holguín-Veras, J., 2008a. Investigation of attributes determining trip chaining behavior in hybrid microsimulation urban freight models. Transportation Research Record 2066, 1-8.

Wang, Q., Holguín-Veras, J., 2008. Tour-based entropy maximization formulations of urban commercial vehicle movements. In: 2009 Annual Meeting of the Transportation Research Board. CDROM.

Wang, Q., Holguín-Veras, J., 2009. Tour-based entropy maximization formulations of urban commercial vehicle movements. In: 2009 Annual Meeting of the Transportation Research Board. CDROM.

Willumsen, L.G., 1978. OD matrices from network data: a comparison of alternative methods for their estimation. In: PTRC Annual Meeting, London, England.

Wilson, A.G., 1967. A statistical theory of spatial distribution models. Transportation Research 1 (3), $253-269$.

Wilson, A.G., 1969a. The use of entropy maximising models in the theory of trip distribution, mode split. Journal of Transport Economics and Policy, 108 126.

Wilson, A.G., 1969b. The use of entropy maximising models in the theory of trip distribution, mode split and route split. Journal of Transport Economics and Policy, 108-126.

Wilson, A.G., 1970. Inter-regional commodity flows: entropy maximizing approaches. Geographical analysis 2 (3), $255-282$.

Wisetjindawat, W., Sano, K., Matsumoto, S., Raothanachonkun, P., 2007. Micro-simulation model for modeling freight agents interactions in urban freight movement. In: 86th Annual Meeting of the Transportation Research Board Washington, DC.

Xu, N., Holguín-Veras, J., 2008. A dynamic model of integrated production-transportation operations. In: Annual Meeting of the Transportation Research Board.

Yang, H., Sasaki, T., Iida, Y., Asakura, Y., 1992. Estimation of origin-destination matrices from link traffic counts on congested networks. Transportation Research Part B 26 (6), 417-434.

Yang, X., Sun, Z., Ban, X.J., Wojtowicz, J., 2014. Urban Freight Performance Evaluation Using GPS Data. Annual Meeting, Washington. 